\title{
New laws of simultaneous contrast?
}

\author{
Vebjørn Ekroll* \\ Institut für Psychologie, Universität Kiel \\ Franz Faul \\ Institut für Psychologie, Universität Kiel
}

November 25, 2011

\begin{abstract}
Drawing on many seemingly disparate and unrelated lines of evidence, we argue that the direction of the simultaneous contrast effect in three-dimensional colour space is given by the difference vector between target and surround ('direction hypothesis'). This challenges the traditional idea according to which the direction of the simultaneous contrast is complementary to the colour of the surround ('complementarity law'). We also argue that the size of the simultaneous contrast effect is either constant or decreases with the difference between target and surround in three-dimensional colour space. The latter proposal challenges Kirschmann's fourth law. Within our theoretical framework, the universally presumed validity of the complementarity law and Kirschmann's fourth law can be understood as resulting from the failure to take various confounding factors into account when interpreting empirical data, the most prominent of which is the influence of temporal von Kries adaptation.
\end{abstract}

Note: This is a preprint of a manuscript accepted for publication in Seeing and Perceiving.

\section{Introduction}

Simultaneous colour contrast is often thought to obey quantitative laws grossly similar to those governing temporal adaptation (or 'successive contrast'). One of these is the 'complementarity law', according to

*vekroll@psychologie.uni-kiel.de 
which the direction of the simultaneous contrast effect is roughly complementary to the colour of the surround. Another one is Kirschmann's fourth law, according to which the size of the simultaneous contrast effect increases with the saturation of the surround (Kirschmann, 1891). In the present theoretical note we shall argue that when the confounding influence of temporal adaptation is taken into account, the effect attributable to pure simultaneous contrast (i.e. the local spatial mechanism) actually obeys other principles. More specifically, we shall argue that the 'complementarity law' should be replaced by the following formulation:

Direction hypothesis: The direction of the simultaneous contrast effect in three-dimensional colour space is given by the vector pointing from target to surround.

The basic difference between the traditional 'complementarity law' and the direction hypothesis is illustrated schematically in Fig. 1.

According to the 'complementarity law', the direction of the simultaneous contrast effect, represented by the arrows in Fig. 1 depends only on the colour of the surround, not on the colour of the target. Thus, when the surround colour is fixed, the direction of the simultaneous contrast effect should be the same for all possible colours of the central targets. According to the proposed 'direction hypothesis', in contrast, the direction of the effect depends just as much on the colour of the target as on the colour of the surround. Thus, even though the surround colour is fixed, the effect can assume any direction in colour space, depending on the colour of the target. Note, however, that the 'complementarity law' and the 'direction hypothesis' make identical predictions in some important cases: If the surround is coloured, the two candidate laws make identical predictions for targets which are a) complementary to the surround, b) nominally grey or c) of the same hue as the surround but less saturated. This is important to note because these constraints are often met in demonstrations and experiments cited as evidence for the complementarity law. In the typical textbook demonstration of simultaneous colour contrast, for instance, in which two identical grey targets are embedded in two complementary surrounds, the proposed direction hypothesis makes exactly the same prediction as the traditional 'complementarity law'. Note also that the proposed direction hypothesis is formulated in terms of threedimensional colour vectors rather than just the two-dimensional chromaticity vectors used for illustration in Fig. 1b.

With regard to the size of the simultaneous contrast effect, we shall question the general validity of Kirschmann's fourth law and contrast it with two alternative hypotheses. The most radical one, in the sense that it is more strongly at odds with Kirschmann's fourth law, is the following 
a

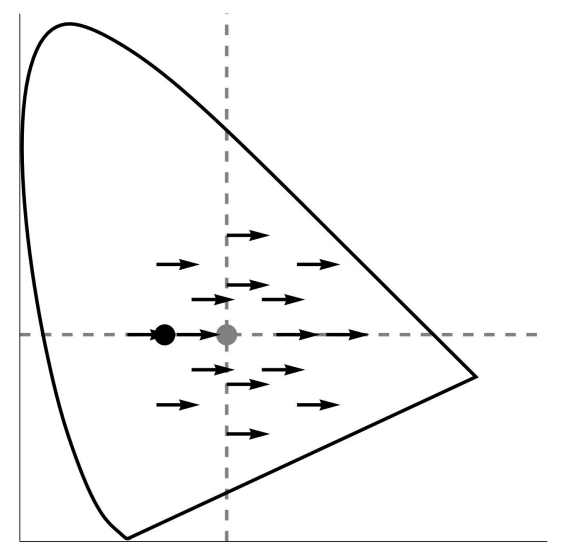

C

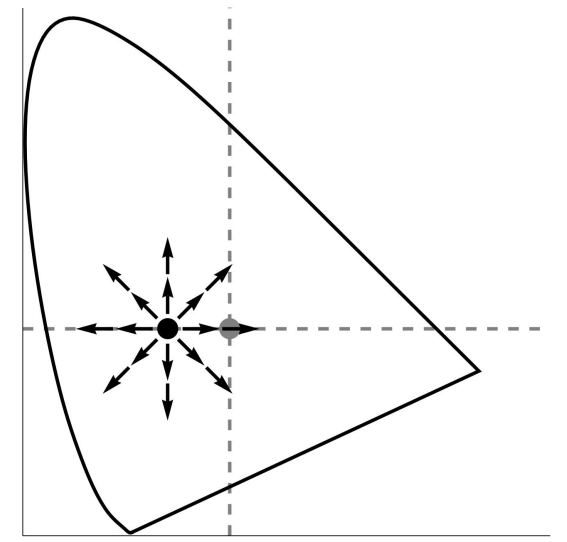

b
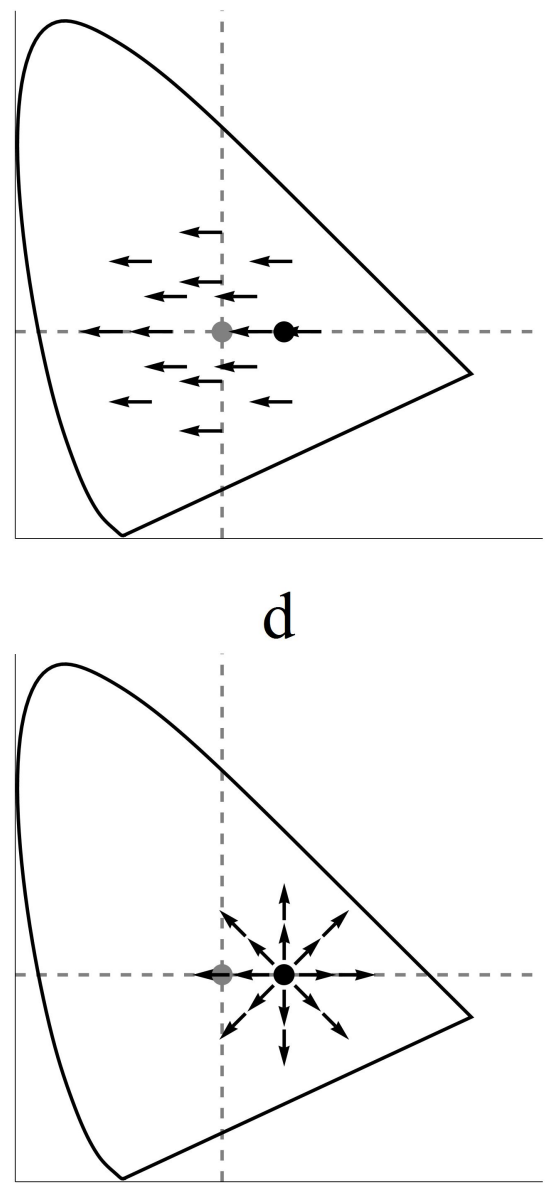

Figure 1: Schematic illustration of simultaneous contrast effects predicted by the traditional complementarity law (top) and the proposed direction hypothesis (bottom). In all panels, the grey dot represents the chromaticity of the nominal grey point, the black dot represents the chromaticity of the surround and the arrows represent the simultaneous contrast effect. Each arrow points from the nominal colour to the perceived one. According to the complementarity law, the shift is always opposite to the vector from the grey point to the surround, and independent of the target chromaticity (Panels a and b). According to the proposed direction law, however, the shift has the same direction as the vector from the surround colour to the target colour (Panels c and d). 
Inverse size hypothesis: The size of the simultaneous colour (i.e. chromaticity and brightness) contrast effect is inversely related to the distance between target and surround in three dimensional-colour space.

The more conservative one is the

Constant size hypothesis: The size of the simultaneous colour (i.e. chromaticity and brightness) contrast effect is essentially constant

According to the latter hypothesis, the size of the effect is independent both of the saturation of the surround and the distance between target and surround in three-dimensional colour space. The empirical evidence presently available is ambiguous with regard to the relative merit of the inverse size and the constant size hypothesis. Thus, rather than making any final commitment to either of these two hypotheses, we shall merely argue that either of them is true. Note that with 'size of the simultaneous contrast effect' we mean distance in three dimensional colour space along the direction specified by the direction hypothesis.

Thus, in the present note, we make two definite claims: a) the 'complementarity law' should be replaced by the direction hypothesis and b) Kirschmann's fourth law should be replaced by either the constant size or the inverse size hypothesis. Our arguments in favour of these claims are based on many seemingly disparate and unrelated lines of evidence and recent developments in our understanding of simultaneous colour contrast (Brown \& MacLeod, 1997; Gordon \& Shapley, 2006; Hansen, Walter, \& Gegenfurtner, 2007; Ekroll, Faul, Niederée, \& Richter, 2002; Ekroll, Faul, \& Niederée, 2004; Ekroll \& Faul, 2009; Ekroll, Faul, \& Wendt, 2011; Faul, Ekroll, \& Wendt, 2008). The main point we wish to make is that many different and seemingly unrelated empirical findings which have accumulated over the years and all appear surprising and challenging from the the traditional point of view can be reinterpreted as converging evidence for the simple principles laid out in the above hypotheses.

Note that together with the direction hypothesis, the constant size hypothesis has a rather interesting corollary:

Step discontinuity corollary: The function relating the nominal cone-excitation vector of the target to colour appearance exhibits a step discontinuity at the point where the cone-excitation vector of the target equals that of the surround

This is illustrated in Fig. 2a. It is easy to see that this corollary remains valid if the constant size hypothesis is replaced by the inverse size hypothesis (Fig. 2b). 

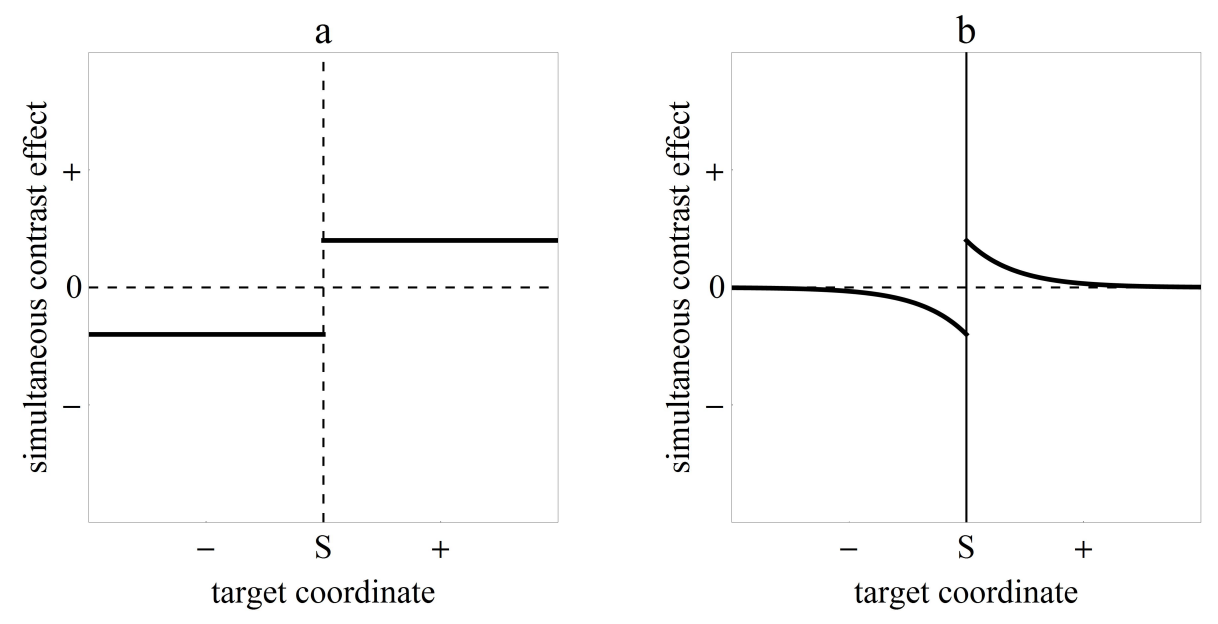

Figure 2: a) If the simultaneous contrast effect is of constant size, but its sign reverses with the sign of the difference between target and surround, a step discontinuity at the coordinates of the surround is implied. This conclusion about scalar values also holds for vectors (where 'direction' rather than 'sign' is the appropriate term). b) A discontinuity is also implied if the simultaneous contrast effect decreases with the difference between target and surround. 
The proposed hypotheses are intended to hold for targets embedded in uniform surrounds which are also discriminably different from the surround itself. In the singular case that the target is equal to (or below the threshold for discriminability against) the uniform surround we propose that there is no simultaneous contrast effect at all. Generally, we assume that in unstructured variegated surrounds, there is no simultaneous contrast effect, provided that the colour variance in the surround is sufficiently large. Thus, different from the classical assumption that a uniform achromatic surround is neutral in the sense that it produces no simultaneous contrast we assume that no uniform surround whatsoever is neutral. Rather, unstructured variegated surrounds of sufficiently high colour variance are assumed to be neutral (with respect to simultaneous contrast, but not with respect to temporal adaptation).

Before we consider the empirical evidence for the proposed hypotheses, two further specifications are in order. The first one is necessary because targets embedded in uniform surrounds have been noted to assume a dual colour impression reminiscent of perceptual transparency (Metelli, 1970), particularly when the contrast between target and surround is low (Brenner \& Cornelissen, 1991; Masin \& Idone, 1981; Brown \& MacLeod, 1997; Mausfeld, 1998; Ekroll et al., 2002, 2004). According to our experience, the target is then perceived as consisting of two layers, whereby one layer is perceived as coextensive with the target region and the other as extending across both the target and the surround. Whenever this kind of perceptual colour scission occurs, the proposed laws are intended to describe the colour appearance of the former layer, not the latter (which is typically perceived to have the same colour as the surround). The second specification is motivated by the possibility that individuals with strongly reduced susceptibility or even immunity to simultaneous contrast may not be all that seldom in the general population (Cataliotti \& Becklen, 2007; Ekroll \& Faul, 2009; Ekroll et al., 2011). The proposed laws can not be expected to hold for individuals lacking the mechanism responsible for simultaneous contrast.

\section{Empirical evidence for the proposed hy- potheses}

We shall now illustrate how the proposed hypotheses and assumptions provide a simple framework for understanding different lines of empirical evidence which appear unrelated, curious or even paradoxical when viewed from the traditional perspective. 


\subsection{The gamut expansion effect}

The most direct empirical evidence for our hypotheses is provided by Brown and MacLeod's (1997) gamut expansion effect (see Fig. 3). The discovery of this effect has stirred much interest precisely because it violates traditional assumptions about the major characteristics of simultaneous contrast. A central feature of the effect is that targets appear more saturated when they are embedded in a uniform grey surround than when they are embedded in a variegated surround being also - on average - grey. The increased perceived saturation of the chromatic targets embedded in the uniform surround can be described as an expansion in colour space, hence the name of the effect. The expansion effect is not limited to the chromatic dimensions of colour space, but also extends to the luminance dimension (Brown \& MacLeod, 1997). That is, a whitish target (i.e. a luminance increment) looks more whitish when embedded in the uniform surround than in the variegated one, while a blackish target (a luminance decrement) looks more blackish. Thus, using our assumption that the simultaneous contrast effect occurs in the uniform surround and not in the variegated one, the illusory 'expansion of colour space' observed by (Brown \& MacLeod, 1997) is clearly in agreement with the proposed direction hypothesis. But, how, one might ask, can the assumption that the effect of interest occurs in the uniform surround and not in the variegated one be justified? Our arguments run as follows: If the effect of interest occurs in the uniform surround rather than the variegated one, then it should be possible to replace the variegated surround without loosing the effect. This is indeed the case. If the variegated surround is replaced by a uniform black or uniform white surround, essentially the same effect can be observed (Faul et al., 2008). Second, adding a thin black border around the targets in the uniform grey surround abolishes the effect almost entirely, which also suggests that the effect of interest is a property of the uniform surround rather than the variegated one (Faul et al., 2008). Third, as the simultaneous contrast effect is conceived of as a local spatial effect, one would expect that in matching experiments where both surrounds are uniform and equal in the region directly adjacent to the targets, but have different levels of colour variability in more distant regions, no gamut expansion effect should be measurable, because it is equal in both surrounds and thus cancels out. The results of Mizokami and Yaguchi (2010) suggest that this is indeed the case. Fourth, one would expect that the gamut expansion effect should not only be observable using a uniform and a variegated surround, but also by using two (differently coloured) uniform surrounds. This also seems to be the case (Ekroll \& Faul, 2009; Ekroll et al., 2011).

Research on the gamut expansion effect does not only suggest that 

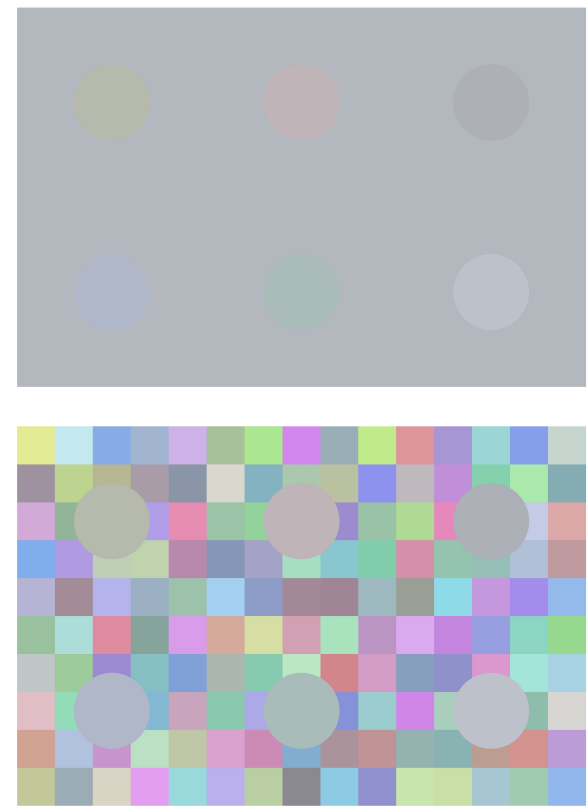

Figure 3: The gamut expansion effect (after Brown and MacLeod, 1997). The six discs embedded in the uniform grey surround are physically identical to those embedded in the variegated ones, but perceptually the colours of the former ones are experienced as more pronounced, i.e. shifted away from the intermediate gray colour of the surround. 
the direction of the effect accords with the direction hypothesis, but also that the size of the effect decreases as the contrast between target and surround increases in accordance with the inverse size hypothesis. Fig. 4 replots pertinent data from Faul et al. (2008). As can be seen, the size of the effect is inversely related to the chromaticity difference $\Delta$ between target and surround.

In summary, the 'gamut expansion effect' that can be observed in Brown and MacLeod's (1997) experimental paradigm accords both with the direction hypothesis and the inverse size hypothesis. A central idea in the present account of simultaneous contrast is the notion that the experimental paradigm introduced by Brown and MacLeod is suitable for observing the pure effects of the simultaneous contrast mechanism. First, since the uniform and the variegated surround have the same mean colour, we assume that any effect of temporal adaptation is identical for both surrounds, such that the differences in colour appearance are due to simultaneous contrast only. Second, since a surround of sufficiently large colour variability is assumed to be neutral with respect to the simultaneous contrast mechanisms, the difference in colour appearance represents a direct measure of the effect occurring in the uniform surround. Traditional experimental paradigms using two uniform surrounds of different colours, on the other hand, can be expected to involve the confounding influence of a temporal adaptation mechanism (Cornelissen \& Brenner, 1991; Ekroll \& Faul, 2009; Ekroll et al., 2011).

\subsection{The 'missing colours' phenomenon}

If, as the evidence from research on the gamut expansion effect suggests, both the direction hypothesis and the inverse size hypothesis are valid, then the above mentioned 'step discontinuity corollary' follows, i.e. the function relating the nominal colour-coordinates of the target to colour appearance should exhibit a step discontinuity at the point where the target is physically equal to the surround. This corollary remains valid also if the inverse size hypothesis is replaced by the more conservative constant size hypothesis. As illustrated in the lower panels of Fig. 5, this means that there is a range of colour impressions - centred around the colour of the surround - that cannot be evoked by any target whatsoever. This gross picture needs to be slightly refined, though, since our hypotheses are only intended to hold for targets which are discriminably different from the surround. In the singular case that the difference between target and surround is zero or below threshold we assume that there is no simultaneous contrast effect. Thus, within the range of missing colours for a given surround (i.e. the vertical range between the two arrowheads in the lower panels of Fig. 5 there is a singular colour that can be evoked, namely that of 

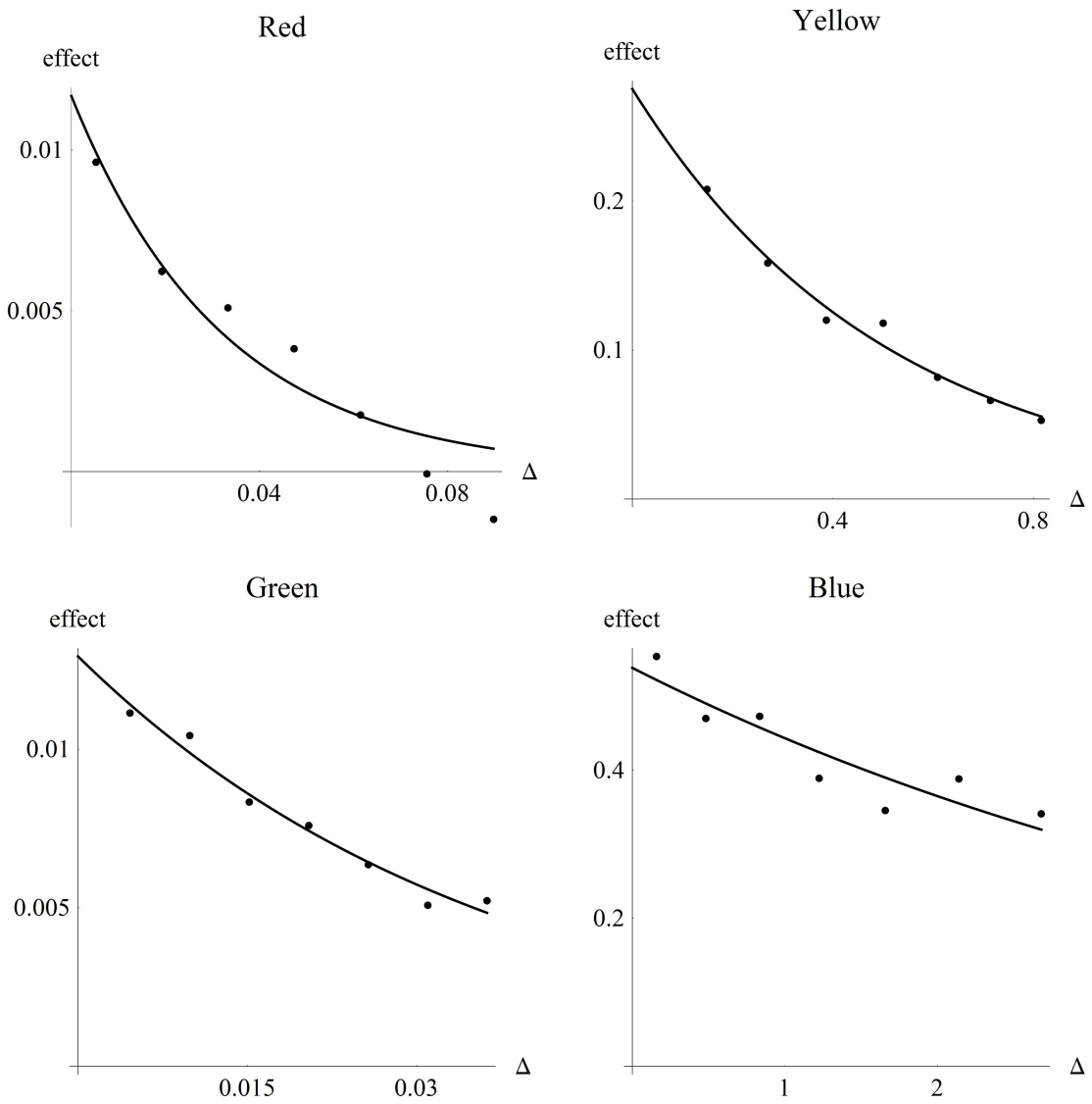

Figure 4: Measurements of the gamut expansion effect replotted from Faul, Ekroll and Wendt (2008, Exp. 1A, Fig. 5). The effect, defined as the difference between the settings of the targets in the variegated surround and the fixed values of the targets in the uniform surround, is plotted against the difference between the fixed target and its uniform grey surround. Each panel shows the data for one of the four half-axes along the $\mathrm{r}$ and $\mathrm{b}$ axes of MacLeod-Boynton (1979) chromaticity space. The function fitted to the data is $f(\Delta):=a e^{-\Delta / b}$. 
the surround (dashed horizontal line).

We have previously reported observations suggesting that such a 'missing colour phenomenon' indeed occurs when uniform surrounds are used (Ekroll et al., 2004). The demonstration in Fig. 6 gives an impression of the effect. Each row displays the same physical scale of coloured squares ranging from blue on the left over grey in the middle to yellow on the right (i.e. the squares are physically identical within each column), yet due to the different surround colours used in each row, the scales appear very different. The scale of colours used for the horizontal background stripes is physically identical to the scale of colours used for the squares, hence the squares in the main diagonal are equal to and thus indistinguishable from the surround. Many observers report seeing all squares above the diagonal as yellowish and all squares below it as bluish. The most interesting observation for present purposes, though, is that the range of yellow appearing targets in the top row include very weakly saturated yellows which are absent in the rows below. Conversely, the range of blue appearing target in the bottom row include weakly saturated blues which are absent in the rows above. In the middle row, both the yellow appearing and the blue appearing parts of the colour scale lacks the most weakly saturated colours. Thus, the colours scale in each row seems to lack a specific range of colour impressions which depends on the colour of the surround. As illustrated in the bottom panels of Fig. 5, this is exactly what one would expect based on our hypotheses: Our hypotheses imply that the range of missing colours should be centred at the colour of the surround. Thus, in a grey surround, desaturated blues and yellows should be missing. As the surround becomes bluer, though, less yellows and more blues should be missing (Fig. 5), and the converse should hold when the surround becomes more yellowish. A presumably related discontinuity of colour appearance has previously been discussed by Whittle (2003, p. 135).

From the above we may conclude that our hypotheses predict surroundspecific ranges of missing colours which are in good qualitative correspondence with empirical observations. To our knowledge, these phenomena of missing colours are not accounted for by any extant model or theory. Using the simplifying approximation that our hypotheses hold for any target colour physically different (rather than perceptually discriminable) from the surround, the region of missing colours predicted by our hypotheses is a sphere in a suitably scaled threedimensional colour space centred at and excluding the colour of the surround. In a two-dimensional plane through the surround colour it is a corresponding circle, and on a one-dimensional line through the surround colour it is a corresponding interval. 

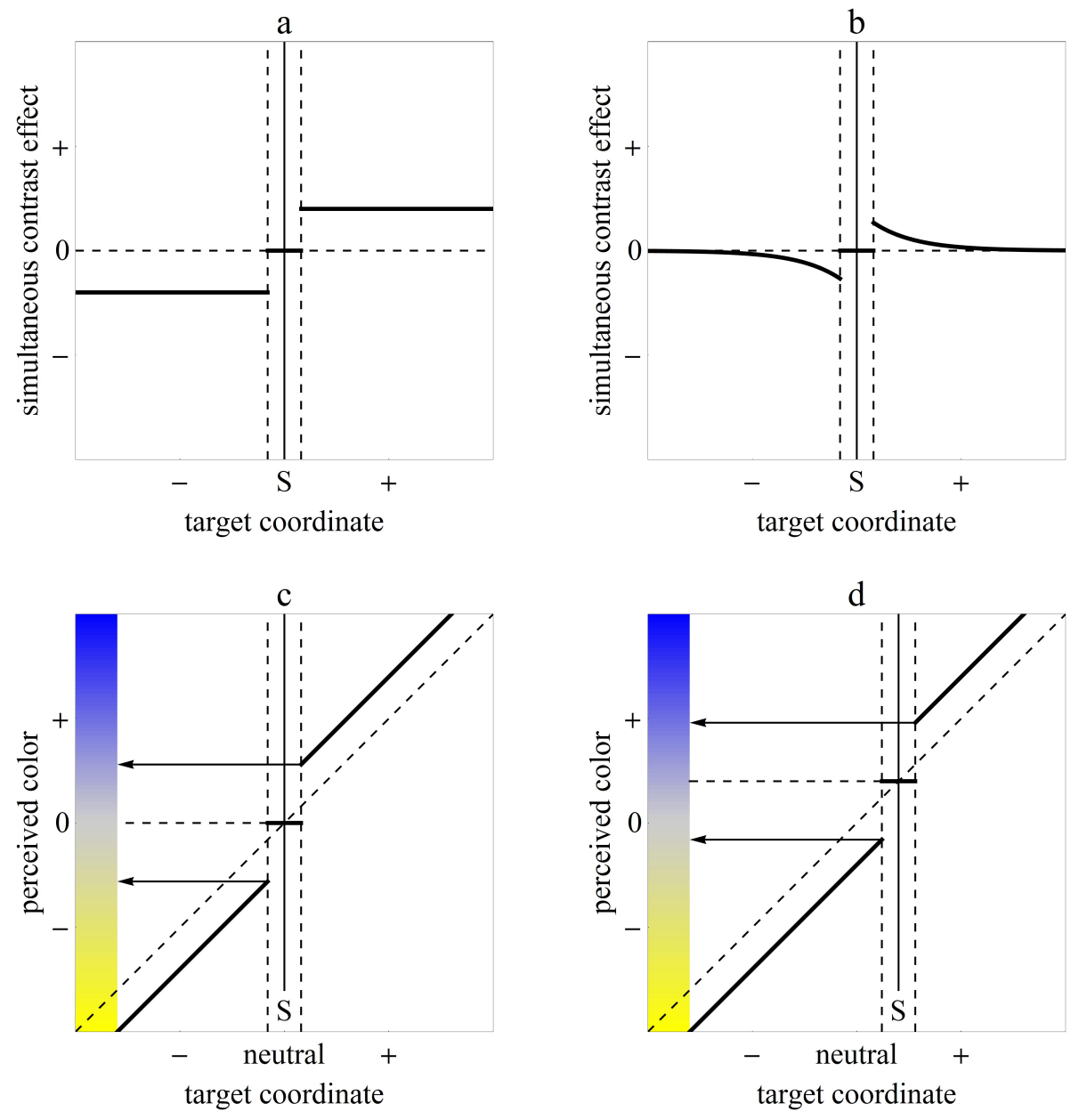

Figure 5: The top panels illustrate the simultaneous contrast effect predicted by the direction hypothesis together with either the constant size hypothesis (a) or the inverse size hypothesis (b). The horizontal axis represents target colours on a line in colour space through the colour of the surround and the vertical axis shows the predicted simultaneous contrast effect (which should occur along the same line in colour space). The solid vertical line indicates the colour of the surround and the dashed vertical lines indicates threshold in each direction. According to our hypotheses, there is no simultaneous contrast effect when the target is below threshold (i.e. indistinguishable from the surround). Panel (c) illustrates how perceived colour should depend on the chromaticity of the target (here along the yellow-blue axis) if the surround is grey (neutral) and the simultaneous contrast effect obeys the direction hypothesis and the constant size hypothesis (as in panel (a)). For suprathreshold targets, the predicted perceived colour is obtained by adding the simultaneous contrast effect in panel a to the nominal chromaticity of the target (dashed diagonal line). Observe how the step discontinuities produce a range of colour impressions (vertical axis, between the arrowheads) which cannot be evoked by any target chromaticity. This iqnge of missing colours is only interrupted by the singular colour impression that can be evoked when the target is equal to the surround (here grey). Panel (d) shows the same as panel (c), but here a bluish rather than a neutral surround is assumed. Note that this results in another range of missing colours including more shades of blue and less shades of yellow. 


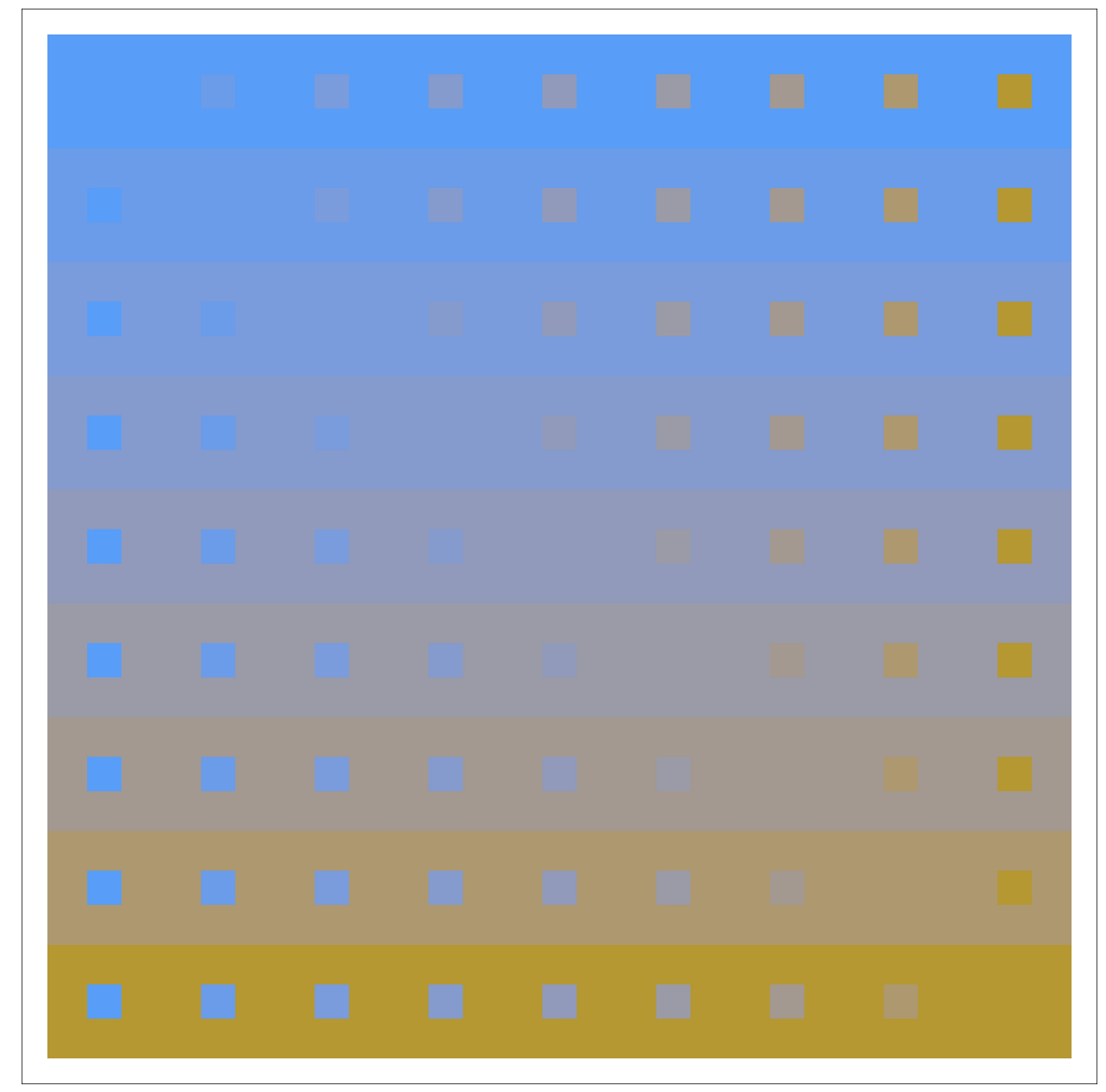

Figure 6: Demonstration of simultaneous contrast and the 'missing colours phenomenon'. The squares are physically identical within each column, but appear rather different. Physically, the colour of the targets in the ith column from the left are identical to that of the ith background stripe from the top. Accordingly, the squares on the main diagonal are identical to the surround and therefore indistinguishable against it. Many observers report that the squares above the main diagonal appears yellowish, while those below it appear bluish, even though the scale of colours used for the targets is the same in each row. Note that the yellowish range of each scale includes more desaturated yellows in the upper rows than in the lower ones. Conversely, the bluish range of each scale includes more desaturated blues in the lower rows than the upper ones. Thus, depending on the colour of the surround, a specific range of colour impressions are missing. Compare with the predictions in the lower panels of Fig. 5. Reproduced from Ekroll (2005). 


\subsection{The convergence paradox and rotation of con- stant hue lines}

The direction hypothesis and the constant size hypothesis (or the inverse size hypothesis) can also account for some further challenging and ill-understood findings, namely the 'convergence paradox' reported in Ekroll et al. (2002) and the rotation of constant hue lines observed by Hansen et al. (2007). Simultaneous contrast is often measured using achromatic settings (Helson \& Michels, 1948; Werner \& Walraven, 1982). In Ekroll et al. (2002), though, we used alternative, indirect methods which - resting on standard assumptions about the topology of the space of perceived colour - should also be suitable for determining the location of the achromatic point. One of these methods was to determine the point at which lines of constant hues converge, which should be the achromatic point. A second method was to determine the smallest hue circle that containes all hues, which should contain the achromatic point within it. The surprising result obtained using these techniques was that the estimated achromatic point did not coincide with a target chromaticity that appeared achromatic but rather with the chromaticity of the coloured surround. That is, lines of constant hue converged on a colour that was coloured rather than achromatic, a result which seem to defy the very definition of constant hue. This 'convergence paradox' was replicated by Hansen et al. (2007) using yet another method. In their study, subjects categorised the colour of targets presented in a coloured surround as having one of eight predefined hues. These judgements were then used to estimate straight hue category boundaries (which should correspond to lines of constant hue) converging at a central point. As in Ekroll et al.'s study, the convergence point turned out to coincide with the chromaticity of the surround rather than a target chromaticity that appeared achromatic. Beyond replicating the convergence paradox, Hansen et al.'s study also revealed that the direction of the estimated lines of constant hue in the chromaticity diagram changed in a systematic way with the chromaticity of the surround. While the angle of hue boundaries with a direction identical or opposite to the direction defined by the hue of the surround remained unchanged, those of intermediate direction were rotated away from the surround direction, whereby hue boundaries with a direction orthogonal to the surround direction rotated most.

We shall now illustrate that both the convergence paradox and the observed rotation of constant hue lines are to be expected if the direction hypothesis and the constant size hypothesis are true. Formally,

these two hypotheses imply that the simultaneous contrast effect $\Delta$ can be written as

$$
\boldsymbol{\Delta}:=\alpha \frac{\mathbf{t}-\mathbf{s}}{\|\mathbf{t}-\mathbf{s}\|}
$$


because then it is a vector having the same direction as the vector from the surround $\mathbf{s}$ to the target $\mathbf{t}$ with a fixed length $\alpha$. The perceived colour of the target is represented by the vector

$$
\mathbf{c}:=\mathbf{t}+\boldsymbol{\Delta}
$$

which is just the sum of the nominal colourimetric vector $\mathbf{t}$ of the target and the simultaneous contrast effect. Hence, in the absence of any simultaneous contrast effect we have the identity $\mathbf{c}=\mathbf{t}$. For simplicity, we consider a idealised colourimetric chromaticity plane parametrised such that, in the absence of any simultaneous contrast effect, the direction and length of the vector $\mathbf{t}=\mathbf{c}$ represent hue and saturation, respectively, i.e. the nominal grey point is located at the origin $(0,0)$. This chromaticity plane is illustrated in the top panels of Fig. 7. Each of these panels shows the set of missing colours for a different surround, that is the set of colour appearance vectors $\mathbf{c}$ for which there does not exist any vector $\mathbf{t} \in \mathbb{R}^{2}$ which is mapped onto it. This set is a disc with radius $\alpha$ centred at the surround colour $\mathbf{s}$ minus the surround colour itself. This is because the simultaneous contrast effect is a displacement of length $\alpha$ in the radial direction away from the surround for all $\mathbf{t} \neq \mathbf{s}$. The panels in the middle row show the chromaticity vectors $\mathbf{t}$ corresponding to the colour appearance vectors on the constant hue lines in the top panels for the three surround conditions. As can be seen, the loci of constant hue converge on the chromaticity of the surround. Thus, the direction hypothesis and the size hypothesis can account for the 'convergence paradox' observed by Ekroll et al. (2002). The loci of constant hue are slightly curved, but if they are approximated by straight lines (dashed lines in the middle panels) the rotations of hue angles observed by Hansen et al. (2007) are evident. The nature of these rotations are most easily visualised in the lower panels, where lines corresponding to the hue angles in the upper and middle panels are drawn using a common origin. Observe that the rotations of the hue directions from the middle panels (central thin lines) relative to the hue directions from the top panels (peripheral thick lines) are all in the direction away from the direction defined by the surround, except for the hue directions corresponding to the direction of the surround and its complementary, which are constant. This is the same pattern of rotations as that observed empirically by Hansen et al. (2007).

The good qualitative correspondence between the pattern of constant hue line rotations predicted by our hypotheses and that observed by Hansen et al. (2007) can be further appreciated by comparing Fig. 8 with their Fig. $6 \mathrm{~b}$. Here, the amount of rotation in the direction away from the direction defined by the surround is plotted against the absolute angular difference between the original constant hue line and the direction defined by the surround. 

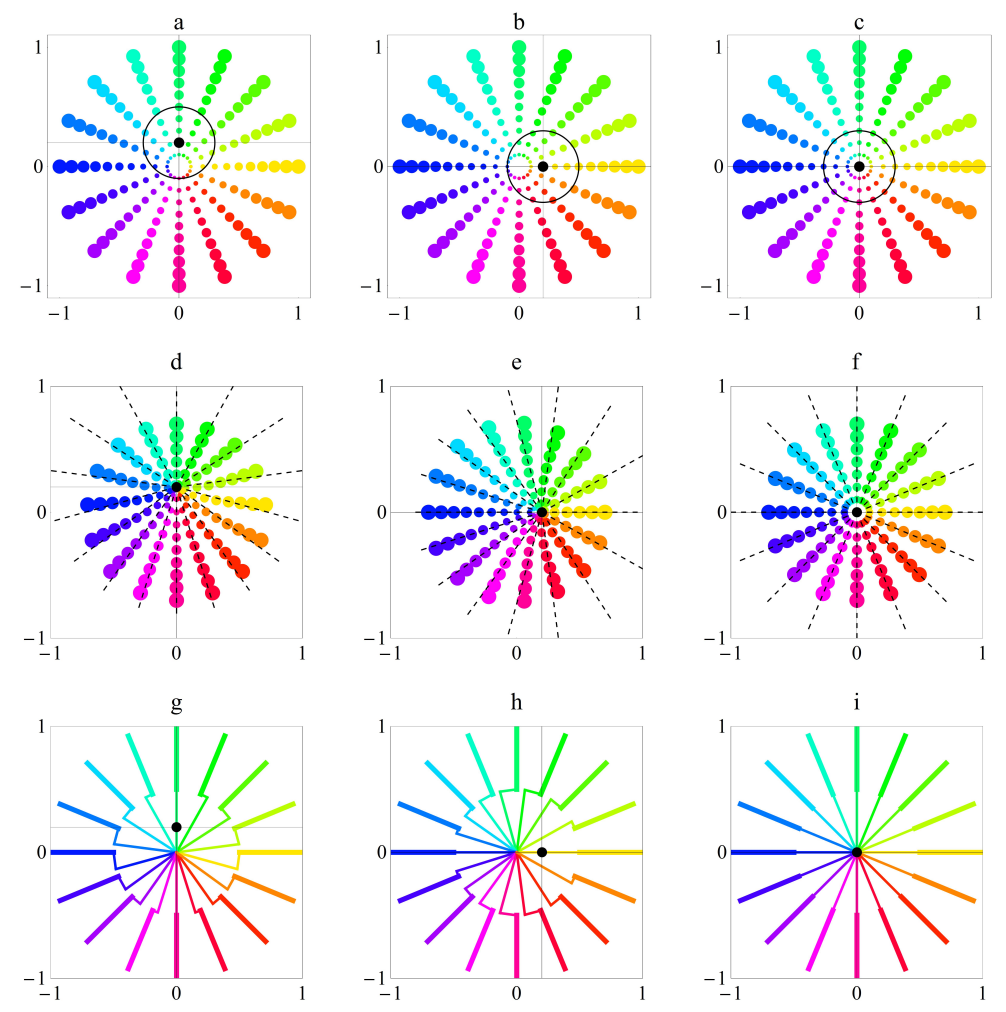

Figure 7: The direction hypothesis together with the constant size hypothesis predict the convergence paradox and rotation of constant hue lines. The three columns each refer to a different surround indicated by the black dot. The left column refers to a green surround with coordinates $(0.2,0)$, the middle one to a yellow surround $(0,0.2)$ and the right one to a grey one $(0,0)$. In all panels, the coordinates of the surround are indicated by a black dot. The upper panels shows the relation between perceived colour and coordinates when there is no simultaneous contrast effect. In this idealised chromaticity space, perceived hue is represented by the hue of the dots and saturation by their size. The achromatic point is located at the origin $(0,0)$. The panels in the middle row show the predicted coordinates of the same perceived colours when they are presented in a uniform surround. The predictions are based on the direction law and the constant size hypothesis (Eqn. 2). Note that loci of constant hue always converge on the colour of the surround (convergence paradox). Note also that the colours within the circles in the upper plots are lacking in the corresponding middle plots. These are the predicted 'missing colours'. When the slightly curved constant hue loci in the middle panels are approximated by straight lines (dashed), then their angles differ from the corresponding angles in the top panels, except when the surround is grey (right column). The bottom panels plot portions of the constant hue lines from the upper and middle plots together and with a common origin to make the rotations of the hue lines more obvious. The outer portions correspond to the upper plots, the inner portions to the middle plots. Note that constant hue lines in the middle plots are rotated in the direction away from the direction corresponding to the $\mathbf{1 6}$ lour of the surround (except in the right row, where the surround is grey). The predictions shown assume that the size of the simultaneous contrast effect ( $\alpha$ in Eqn. 1 ) is 0.3 . This value corresponds to the radius of the circles in the upper plots. 


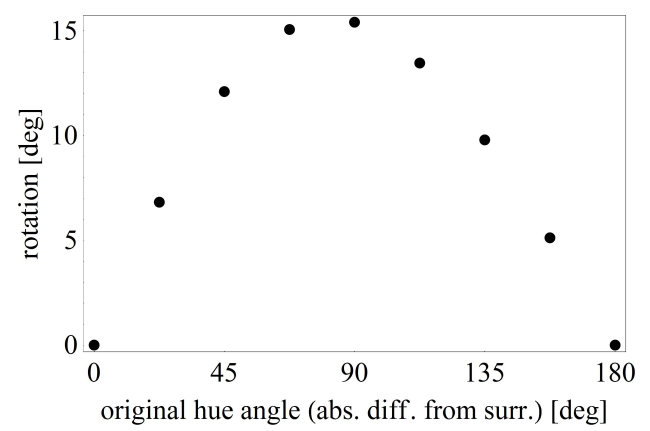

Figure 8: Rotation of the constant hue lines in panels $(\mathrm{g})$ and $(\mathrm{h})$ of Fig. 7. The angle of rotation (in the direction away from the surround colour) is plotted against the angles of the original hue lines. The latter values are given relative to the angle defined by surround colour (hence the predictions are identical for both surrounds). This prediction accords rather well with the data of Hansen et al. (2007, compare with their Fig. 6b).

The predictions shown in Fig. 7 were derived using specific values for the surround chromaticities and the size $\alpha$ of the simultaneous contrast effect. Generally, though, the convergence paradox will be predicted whenever $\alpha$ is larger than the distance from the nominally achromatic origin $(0,0)$ to the surround $\mathbf{s}$, i.e when $\alpha>\|\mathbf{s}\|$. Intuitively, this is because the circle delimiting the set of missing colours in the top panels then intersects all constant hue lines and all points on this circle are all mapped onto the singlar point representing the surround in the middle panels. When the surround is achromatic (right panels in Fig. 7) there is no hue line rotation, but there are missing colours.

In the above, we have demonstrated that the direction hypothesis and the constant size hypothesis together can predict the convergence paradox observed by Ekroll et al. (2002) and the hue line rotation observed by Hansen et al. (2007). Though the argumentation is more complicated, the same main results can also be derived if the constant size hypothesis is replaced by the inverse size hypothesis.

\subsection{Kirschmann's third law}

According to Kirschmann's third law, the simultaneous colour contrast effect is at its strongest when target and surround are equally bright. This effect is not accounted for by dominant models of simultaneous contrast such as the dual process theory of Jameson and Hurvich (1961), yet recent work by Gordon and Shapley (2006) provides striking evidence in favour of its validity. Given that leading 
models fail to account for Kirschmann's third law, Gordon and Shapley's claim that their strong confirmation of it "'require a new theory of colour appearance"' (p. 133) is very much to the point. In this section, we shall illustrate that Kirschmann's third law can be understood as a corollary of the direction hypothesis and the constant size hypothesis (or, alternatively, the inverse size hypothesis). The theoretical interest of this is that it provides a link between Kirschmann's third law and more general principles of broader applicability. Conversely, evidence for Kirschmann's third law may also be considered to constitute additional evidence for our hypotheses.

The argument is very simple, and illustrated in Fig. 9. We consider a colour space with two opponent colour axes (say red-green and blueyellow) and a brightness axis, and refer to targets of different brightness located on the achromatic axis (vertical line in panels a and b), as in Gordon and Shapley's study. We choose an arbitrary coloured surround (large point). Together with the achromatic axis, the surround defines a plane which can be parametrised in terms of one of the opponent colour axes and the brightness axis. The solid arrows in panel a shows the simultaneous contrast effect for the chosen surround (large point) for each of the targets (small points). The direction of the arrows is identical to that of the vector from the surround colour to the nominal target colour (in accordance with the direction hypothesis) and their lengths are identical (in accordance with the constant size hypothesis). Decomposing the vectors representing the simultaneous contrast effects into the brightness components (vertical) and opponent colour components (horizontal), it is evident that the latter is maximal when the brightness of the target is equal to that of the surround. As the brightness difference between the target and the surround increases, the opponent colours component decreases, while the absolute value of the brightness component increases. Panel c refers to the situation depicted in panel a, and shows how the chromatic component (solid curve) of the simultaneous contrast effect should depend on the brightness of the target. The dashed line is Gordon and Shapley's model fitted to our prediction. As can be seen, the models make very similar predictions for the chromatic component of the simultaneous contrast effect. Thus, Gordon and Shapley's model and our hypotheses are equally compatible with their data.

Gordon and Shapley presented their model as an initial working hypothesis tailored to describe their particular findings. Our hypotheses, in contrast, have a broader scope, as they can also account for the findings described in the previous sections. Different from Gordon and Shapley's model our hypotheses also make predictions about the brightness component of the simultaneous contrast effect. The dotted curve in panel $\mathrm{c}$ shows the predicted brightness component of the simultaneous contrast effect for the same surround as the one assumed 
a

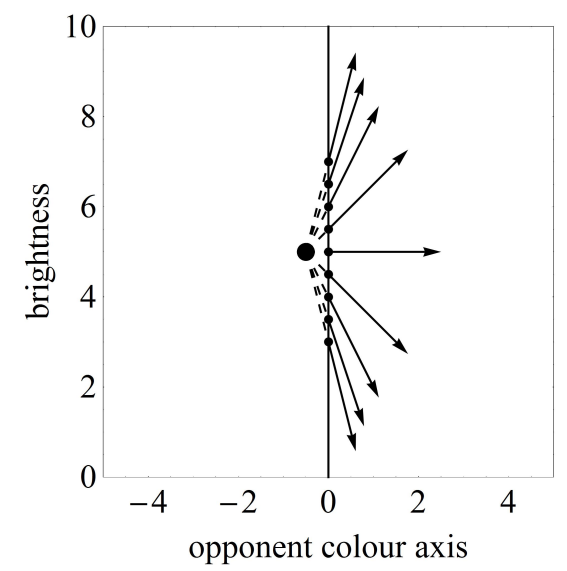

c

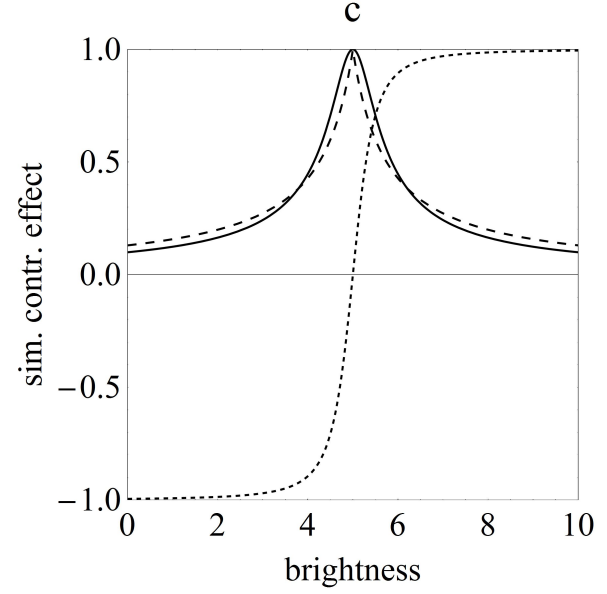

b

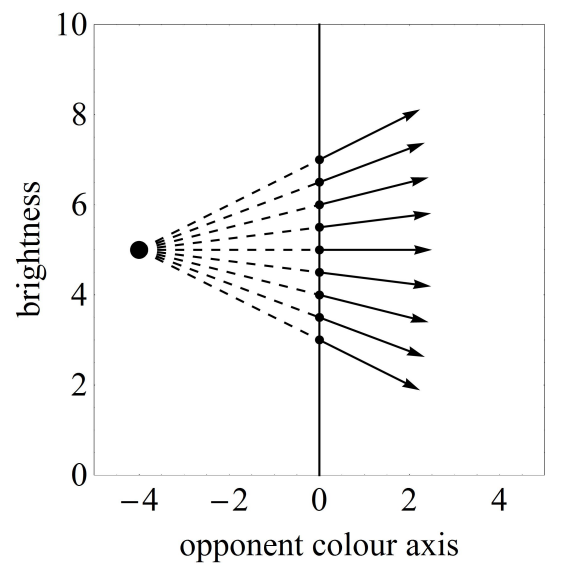

d

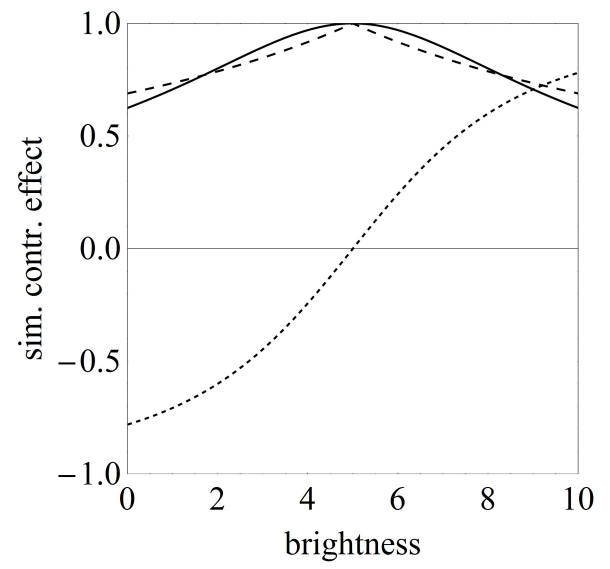

Figure 9: (a) Illustration of why Kirschmann's third law ('chromatic simultaneous contrast is maximal when target and surround are equally bright') follows from the direction hypothesis and the constant size hypothesis. Small dots represent different nominally grey targets in a coloured surround (large dot). The predicted simultaneous contrast effects are represented by the solid arrows attached to the points representing the targets. The length of the arrows are all equal (equal size hypothesis) and their direction correspond to that of the vector from surround to target (direction hypothesis). As can be seen, the chromatic component of the effect (projection of the arrows onto the horizontal axis) is largest for the target which has the same brightness as the surround. Panel (b) shows same as panel (a), but for a more saturated surround. In this case, the chromatic component of the simultaneous contrast effect should decrease more slowly with the (absolute) brightness difference between target and surround. Panel (c) shows how the chromatic simultaneous contrast (solid curve) should depend on the brightness of the target for the same surround as in (a). The dotted curve shows corresponding predictions for the brightness component of the effect (projection of the arrows in (a) onto the vertical axis). The dashed curve shows (oordon and Shapley's (2006) model fitted to our prediction of the chromatic effect. Note that the difference in the predictions is but very slight. Panel (d) shows the same as panel (c), but referring to the more saturated surround shown in panel (b). 
in panel a. As can be seen the brightness effect should have the same sign as the brightness difference between target and surround, change rapidly in the brightness range close to the surround and asymptote at a maximal level as the absolute target-surround differences becomes larger. Our hypotheses predicts that when the saturation of the surround is increased, the chromatic and brightness components of the simultaneous contrast effect should change less rapidly with the brightness of the target. This can be appreciated by comparing the left-hand and right-hand panels in Fig. 9. The demonstration shown in Fig. 10 suggest that these predictions of our hypotheses are indeed borne out. The same physical intensity scale of nominally achromatic targets is reproduced in all five columns. Observe that the range of physical intensities (vertical range) over which a notable chromatic contrast effect can be observed is very small for the targets in the most desaturated coloured surround (second row from the left) and much larger for the most saturated surround. Qualitatively, these empirical observations accord well with the sharp and broad prediction curves (solid curves) in the lower panels of Fig. 9. Observe also that the perceived brightness changes sharply at the background level in the most desaturated surround but much more gradually in the most saturated surround. These observations accord with the dotted prediction curves in the lower panels of Fig. 9. In summary, Kirschmann's third law can be understood as a special case of the direction hypothesis together with the constant size hypothesis. Essentially the same conclusions can be drawn if the constant size hypothesis is replaced by the more radical inverse size hypothesis.

\section{The interplay between simultaneous con- trast and temporal adaptation}

In most situations, the perceived colour of a target will not only be influenced by simultaneous contrast, but also by temporal adaptation (Cornelissen \& Brenner, 1991). Thus, the empirical plausibility of our hypotheses about simultaneous contrast must be evaluated in conjunction with assumptions about the characteristics of temporal adaptation and how it interacts with simultaneous contrast. Our hypotheses were developed with the following assumptions about the influence of temporal adaptation in mind: First, we assume that the temporal adaptation occurs at a stage in the visual process prior to that responsible for simultaneous contrast. Second, we assume that temporal adaptation can be described as von Kries gain control (Kries, 1905). Specifically, if $\mathbf{t}:=\left(t_{l}, t_{m}, t_{s}\right)$ is the nominal cone excitation vector of the target, then the effect of adaptation can be described by multiplying each of the cone excitation values $t_{i}$ with corresponding gain factors $\rho_{i} \leq 1$ 

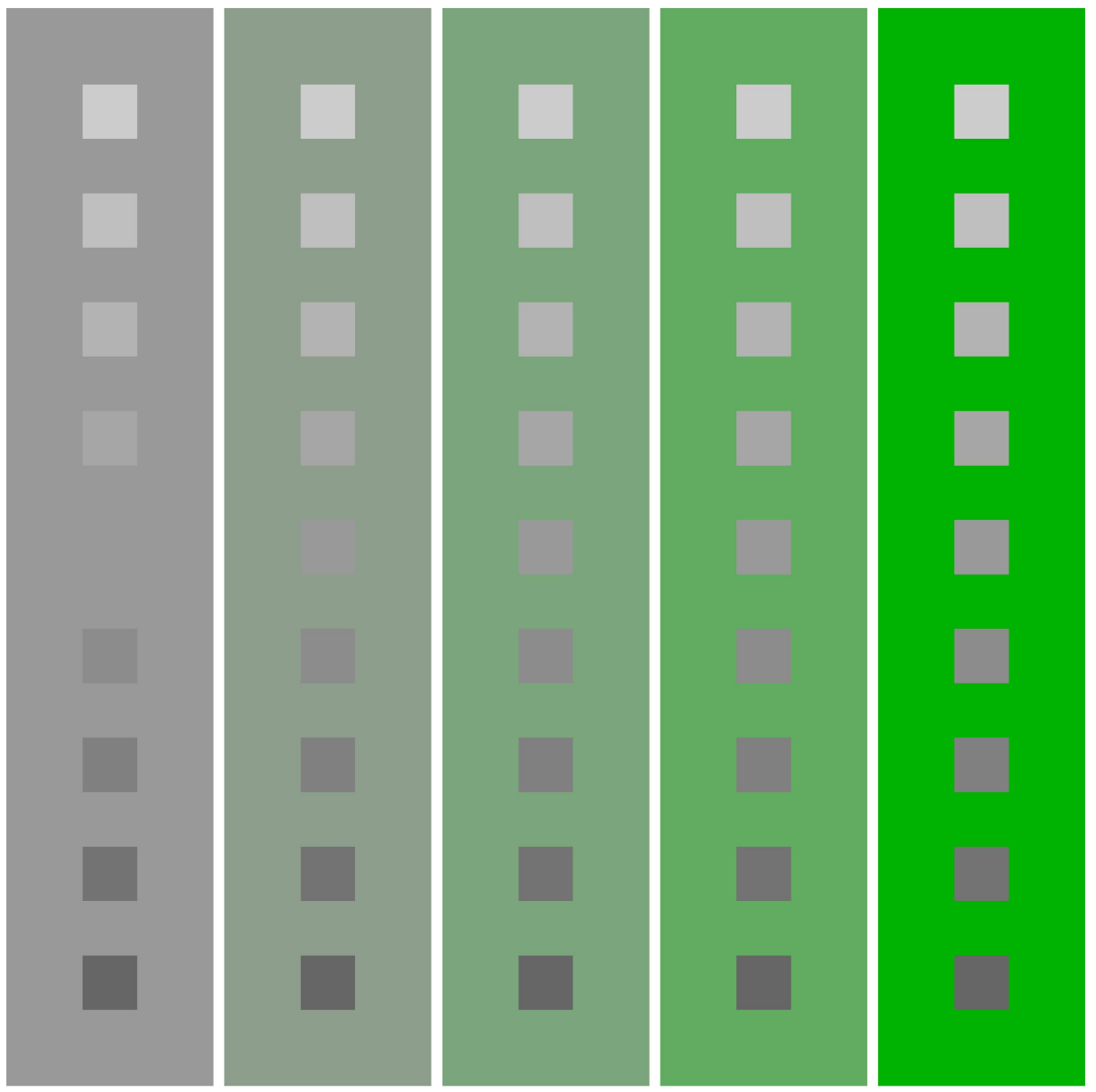

Figure 10: Five physically identical intensity scales presented in surrounds of different saturation (zero on the left and increasing towards the right). Observe that the range of physical intensities (vertical range) over which a notable chromatic contrast effect can be observed is very small for the targets in the most desaturated coloured surround (second column from the left) and much larger for the most saturated surround. Note also that the perceived brightness changes sharply at the background level in the most desaturated surround but much more gradually in the most saturated one. Both of theses observations are predicted by our hypotheses (compare with predictions in the bottom panels of Fig. 9 and see text for details). 
which are inversely related to the corresponding cone-excitation values $a_{i}$ of the adapting stimulus. In the absence of any adaptation, $\rho_{i}=1$ and adaptation is said to be complete when $\rho_{i}=1 / a_{i}$. In the latter case, the adaptation is so strong that any two adapting stimuli $\mathbf{a}, \mathbf{b}$, despite being physically different, are equivalent after adaptation (because then $\rho_{i} a_{i}=a_{i} / a_{i}=b_{i} / b_{i}=\rho_{i} b_{i}$ ). Further, we assume that temporal adaptation depends only on the mean colour of the adapting stimulus. That is, a variegated surround will have the same adapting effect as a uniform one provided that it has the same mean colour (Valberg \& Lange-Malecki, 1990). In the case of centre-surround stimuli, both the target and the surround can be expected to influence the adaptive state. For simplicity, though, we identify the adaptive stimulus with the surround, since the area of the surround tends to be much larger than that of the target.

Resting on these assumptions, we shall now consider what experimental conditions are most suitable for testing the validity of the proposed hypotheses. Obviously, conditions under which the influence of von Kries adaptation is absent or negligible would yield the most diagnostic results. Realising such conditions by limiting viewing time is not easy, since a significant amount of temporal adaptation occurs already after 40-70 ms (Rinner \& Gegenfurtner, 2000). That is, even when the time used to make a judgement or setting is very brief, it is difficult to rule out a non-negligible contribution of temporal adaptation. Thus, a better possibility to eliminate the confounding effect of temporal adaptation in asymmetric matching experiments would be to use two surrounds which have the same adapting influence regardless of inspection time, as in Brown and MacLeod's (1997) study. Accordingly, we regard their experimental set-up as the best possibility for measuring the simultaneous contrast effect directly. A further important corollary of our assumptions is that - quite contrary to widespread assumptions - asymmetric matching experiments under conditions when temporal adaptation is complete yields absolutely no information about the simultaneous contrast effect at all. The simple reason for this is that once adaptation is complete, the two physically different surrounds are identical at the level feeding into the mechanism responsible for simultaneous contrast. Therefore, for the same reasons as an asymmetric matching experiment with physically identical surrounds does not yield any information about the simultaneous contrast effect, this kind of experiment also fails to do so. Note that this does not mean that the simultaneous contrast effect is in any way absent: it just cancels out in the matching equation. Asymmetric matching experiments under conditions where adaptation can be expected to be complete or nearly so have been used quite frequently in the literature (e.g. Whittle \& Challands, 1969; Whittle, 1994; Chichilnisky \& Wandell, 1995; Wuerger, 1996; Niederée \& Mausfeld, 1997; Shepherd, 1997, 
1999; Richter, 2002). Based on the above reasoning the results of these experiments can be taken to demonstrate the dramatic consequences of temporal adaptation, but little, if anything about the characteristic features of simultaneous contrast.

Whittle (1994) has noted that establishing a subjectively satisfactory match in asymmetric matching experiments, which is sometimes experienced as quite difficult when side-by-side displays are used (Gelb, 1929; Burgh \& Grindley, 1962; Ekroll et al., 2004; Vladusich, Lucassen, \& Cornelissen, 2007) is experienced as easy and unproblematic when haploscopically superimposed displays are used. Based on our hypotheses, this curious observation is actually to be expected. As illustrated in the bottom panels of Fig. 5 different surrounds produce different sets of missing colours, which explains why finding a true colour match in asymmetric colour matching experiments is sometimes impossible. If adaptation is complete, though, as it can be expected to be in typical experiments using haploscopically superimposed displays, the sets of missing colours (and the respective complementary sets of realisable colors) are identical in both surrounds. This is because the two sets of missing colours are spheres with the same radius centred at the coordinates of the surrounds, which are identical when adaptation is complete. Thus, in conjunction with the above assumptions our hypotheses about simultaneous contrast can explain not only the subjective difficulties with asymmetric matching in side-by-side displays, but also their absence in haploscopically superimposed displays. The critical variable, though, is not side-by-side vs haploscopically superimposed viewing, but rather the amount of adaptation. Thus, one would expect that the subjective matching problems can also be abolished in side-by-side displays, provided that other conditions such as strict fixation and prolonged viewing of the background are favourable towards producing complete adaptation. Informal preliminary observations suggests that this is indeed the case.

It is important to note that von Kries adaptation will tend to produce results that are in accordance with the traditional 'complementarity law' and Kirschmann's fourth law. Therefore, results of studies where adaptation play a non-negligible role may accord with the complementarity law and Kirschmann's fourth law even though the simultaneous contrast effect itself actually does not.

\section{Kirschmann's fourth law vs. the con- stant and inverse size hypotheses}

According to Kirschmann's fourth law, the chromatic simultaneous contrast effect increases with the saturation of the surround. In actual 
research practice, though, the main evidence for this law stems from studies in which the target was nominally achromatic (Kirschmann, 1891; Kinney, 1962; Valberg, 1974; Cornelissen \& Brenner, 1991; Bosten \& Mollon, 2007). In this case, it is unclear whether it is actually the saturation of the surround, or the difference between target and surround which is the critical variable. Reformulating Kirschmann's fourth law in terms of contrast, it would state that simultaneous contrast increases with the difference between target and surround. Our constant size hypothesis denies that there is such an increase, and our inverse size hypothesis states that the effect even decreases. Which of these three alternatives is most plausible in light of the available empirical evidence? The relevant evidence is arguably rather diverging, suggesting that different potentially confounding factors must be taken into account in order to arrive at conclusions of more general validity. The main evidence for the inverse size law stems from our study of the gamut expansion effect (Faul et al., 2008). In this case, a confounding influence of temporal adaptation is highly unlikely, since both surrounds have the same mean colour. It can be questioned, though, whether the inverse relationship between the target-surround difference and the size of the effect observed in this study generalises to surrounds which are coloured rather than achromatic. A second line of evidence for the inverse contrast hypothesis stems from reports pertaining to the phenomenon interchangeably referred to as 'Florkontrast', 'tissue contrast', 'Gauzkontrast' or 'Meyer's effect' (Meyer, 1855; Hering, 1887; Helmholtz, 1911; Köhler, 1904; Perls, 1932; Hildreth, 1949; Walls, 1960; Brown, 2003; Mausfeld, 2003). The basic observation referred to here, is that simultaneous contrast effects can be enhanced by covering the stimulus with tissue paper, or diluting it with white light, both of which should tend to reduce the contrast between target and surround (as well as the saturation of the surround). The bulk of these reports refer to informal observations and demonstrations rather than formal experiments, though.

Several more formal experiments have investigated how colour induced into a nominally neutral target depends on the saturation of the surround (Kirschmann, 1891; Kinney, 1962; Valberg, 1974; Cornelissen \& Brenner, 1991; Bosten \& Mollon, 2007). Before we discuss the mixed findings from these studies in more detail, though, we shall consider some potential caveats which need to be considered when evaluating them. These considerations may also be of value for devising future experiments aiming to resolve the issue more conclusively.

Caveat A: The influence of adaptation Temporal von Kries adaptation alone will produce results in accordance with Kirschmann's fourth law. Thus, even if the constant size hypothesis or the inverse hypothesis is valid, the combined, directly observable effect may still 
increase with the difference between target and surround. If the constant size hypothesis is true, any contribution of adaptation, however slight, will lead to a (correspondingly slight) increase of the observable effect with the difference between target and surround. If the inverse size hypothesis is true, then larger amounts of adaptation will be necessary to obtain an increasing observable effect. Note, however, that the pure simultaneous contrast effect, even under optimal conditions, is presumably rather small in absolute terms: All putative observations of very large simultaneous contrast known to us involve conditions under which temporal adaptation can be expected to contribute considerably to the observable effect. Under conditions where this is clearly not the case, as in the gamut expansion effect (Brown \& MacLeod, 1997; Faul et al., 2008), the simultaneous contrast effect is rather modest when measured in absolute terms. Thus, even if the inverse size hypothesis is true, moderate amounts of adaptation may suffice to yield a combined observable effect that increases with the difference between target and surround.

Caveat B: Target-surround differences below or near threshold Consider the following experimental situation, realised for instance in the studies of Cornelissen and Brenner (1991) and Bosten and Mollon (2007): The target is achromatic and embedded in different surrounds all equiluminant to the target but of different saturations (or, equivalently, chromatic contrasts relative to the target). From a traditional perspective, one would expect the simultaneous contrast effect to be adequately represented by a continous function on the domain of all possible target-surround contrasts, including zero, and based on Kirschmann's fourth law, this function should be monotonically increasing. Based on our hypotheses, however, the effect should - in theory - exhibit a step discontinuity at the target-surround contrast at which the surround becomes discriminably different from the target. From the traditional perspective, it is hardly relevant whether the target-surround contrast is just above or just below threshold, because the simultaneous contrast effect can be expected to be very small anyway. From our perspective, though, this should make all the difference. This is because the effect should be zero when the surround is perceptually identical to the surround, but reach its maximal value as soon as the surround is perceived as different from the surround. At still larger target-surround contrasts, the effect should remain at its maximum if the constant size hypothesis is presupposed or decrease again if the inverse size hypothesis is assumed to be true. As illustrated in Fig. 11, though, even if the constant size (panel a) or the inverse size hypothesis (panel b) is valid, the sharp step discontinuity implied cannot be expected to be manifest in empirical data averaged across repeated trials (or observers). This is because of the probabilis- 

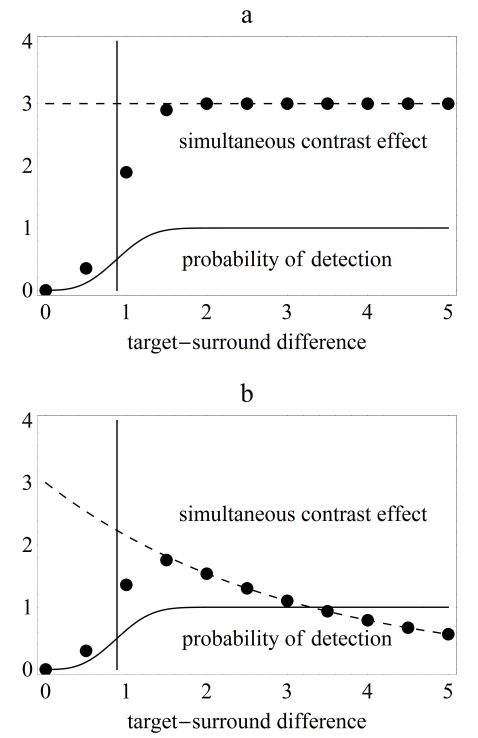

Figure 11: Panel (a): Illustration of how averaged data points from empirical studies may suggest an increase in the simultaneous contrast effect (vertical axis) with the target-surround difference (horizontal axis) even though the constant size hypothesis (dashed horizontal line) is valid. According to our assumptions, there is no simultaneous contrast effect for targets below threshold discriminability against the surround (left of the solid vertical line). Since the threshold is probabilistic in nature, one needs to consider the probability that the target is discriminated against the surround, indicated by the solid curve. Expected averaged data (black dots) will follow the ideal dashed line multiplied with the solid curve indicating the probability of detection. Panel (b) shows the same as panel (a) but here the constant size hypothesis is replaced with the inverse size hypothesis.

tic nature of discriminability. At a given target-surround contrast in the vicinity of the threshold, the surround will be perceived as different from the target with a certain probability $p$. Let the size of the theoretical simultaneous contrast effect be $e$ for a discriminable surround and remember that it is zero for an indiscriminable surround. Then the expected setting will be $p \cdot e+(1-p) \cdot 0=p \cdot e$. Such expected settings are shown as dots in Fig. 11. As can be seen, the expected settings initially increase as a function of target-surround contrast both in case of the constant size hypotheses and in case of the inverse size hypothesis. These theoretical examples illustrate that in the vicinity of threshold, averaged data may increase with target-surround contrast even though the constant size hypothesis or the inverse contrast hypothesis is true. 
Caveat C: The influence of the comparison surround In the experiments of Cornelissen and Brenner (1991) and Bosten and Mollon (2007), the subjects matched the perceived colour of the nominally grey test target embedded in a coloured test surround by adjusting the chromaticity of a comparison target embedded in a uniform grey comparison surround of the same luminance. From a traditional point of view, this appears convenient, since such a surround may be considered to be 'neutral', that is to produce no simultaneous contrast and to produce a 'normal' state of adaptation. If our hypotheses are valid, however, the use of this particular comparison surround introduces special problems, which may appear surprising but are rather direct corollaries of our hypotheses. Simply stated, the problem is that the perceived colours predicted for the test targets are always members of the set of missing colours for targets in the comparison surround. Thus, the very colour impressions that are to be measured using the matching technique should not be realisible using targets in that particular comparison surround as a probe.

This is illustrated in Fig. 12. Panel (a) shows how perceived colour should depend on the colourimetric coordinates of the target for targets located on a line in colour space from yellow to blue, provided that the surround $S$ is grey (and located on the same line in colour space). The solid vertical line shows the coordinate of the grey surround and the dashed vertical lines represent threshold in each direction. Below threshold, the target appears equal to the surround (solid thick horizontal line). Neglecting this singular case, the grey region indicates the missing colours (along the vertical axis) for this grey surround. Panels (b) and (c) show the predictions for a moderately and a more strongly saturated blue surround, respectively, but note that the grey regions are copied from panel (a) and therefore indicate the missing colours in the grey surround, and not the different sets of missing colours in the blue surrounds. The predicted colour appearance of a neutral target viewed in the blue surrounds in panels (b) and (c) is indicated by arrow (2). Note that the same perceived colour is predicted for both of the blue surrounds and that it is located within the set of missing colours for the grey surround. Thus, when the nominally grey target is presented in either of the blue surrounds, it cannot by matched by any colour that is realisable by any target whatsoever in a grey comparison surround. Confronted with this problem, it is reasonable to expect that subjects choose the closest realisable colour, which is pointed to by arrow (3). Arrows (4) and (5) in panel (a) show which colourimetric coordinate must be chosen for the target in the grey comparison surround in order to produce this colour impression. As can be seen, this is a yellow target which is just discriminable against the grey surround. Thus, if the constant size hypothesis is valid, one would expect subjects to always adjust the colour for the target in the comparison 
surround such that it is complementary to the colour of the surround and just discriminable against the grey comparison surround.

How will this prediction change if the constant size hypothesis is replaced by the inverse size hypothesis? The simple, and perhaps surprising answer is not at all. The bottom panels in Fig. 12 shows the same as the upper panels assuming the inverse size hypothesis rather than the constant size hypothesis. In this case, the yellow perceived colour of the nominally grey target embedded in the blue test surrounds becomes less saturated as the saturation of the blue surround increases, but the closest realisable colour in the grey comparison surround remains the same as in the case of the constant size hypothesis.

Note that these predictions are particular to the special case in which the comparison surround is equal to the test target (in this case a particular grey). If another surround, say, a black one, is used instead the colours to be matched will not fall into the set of missing colours. In this case, the constant size and the inverse size hypothesis predict different matching data just as one would intuitively expect. It is also instructive to note that our hypotheses predict a larger measured effect for a black surround: According to the direction law, the simultaneous contrast effect in the black surround will be mainly in the luminance direction for most targets, and the pure chromatic component of the effect will be correspondingly smaller. Thus, more saturated chromaticites must be chosen for the targets to match the perceived saturation of the test targets.

Caveat D: Brightness differences Consider the above kind of experiment modified such that there is a constant brightness difference between the test target and the test surrounds investigated. Then, if the constant size hypothesis is true, the purely chromatic component of the simultaneous contrast would not be constant, but rather increase with the chromatic difference between target and surround. The reason for this can be gleaned from Fig. 9, panels a and b: As the chromatic difference between target and surround decreases, the vector representing the simultaneous contrast effect rotates, and as its brightness component increases, its chromatic component decreases. Fig. 13a shows predictions based on the constant size hypothesis for three different brightness differences $\Delta B$ between target and surrounds. Panel b shows the same based on the inverse contrast hypothesis.

The essential point illustrated here is that if there is a brightness difference between target and surround, an increase in the observed simultaneous contrast effect with the saturation of the surround will be predicted even though the constant size hypothesis or the inverse size hypothesis rather than Kirschmann's fourth law is valid. To evaluate the validity of our hypotheses in the above kind of experiment, it is therefore essential that the brightness values of target and surrounds 

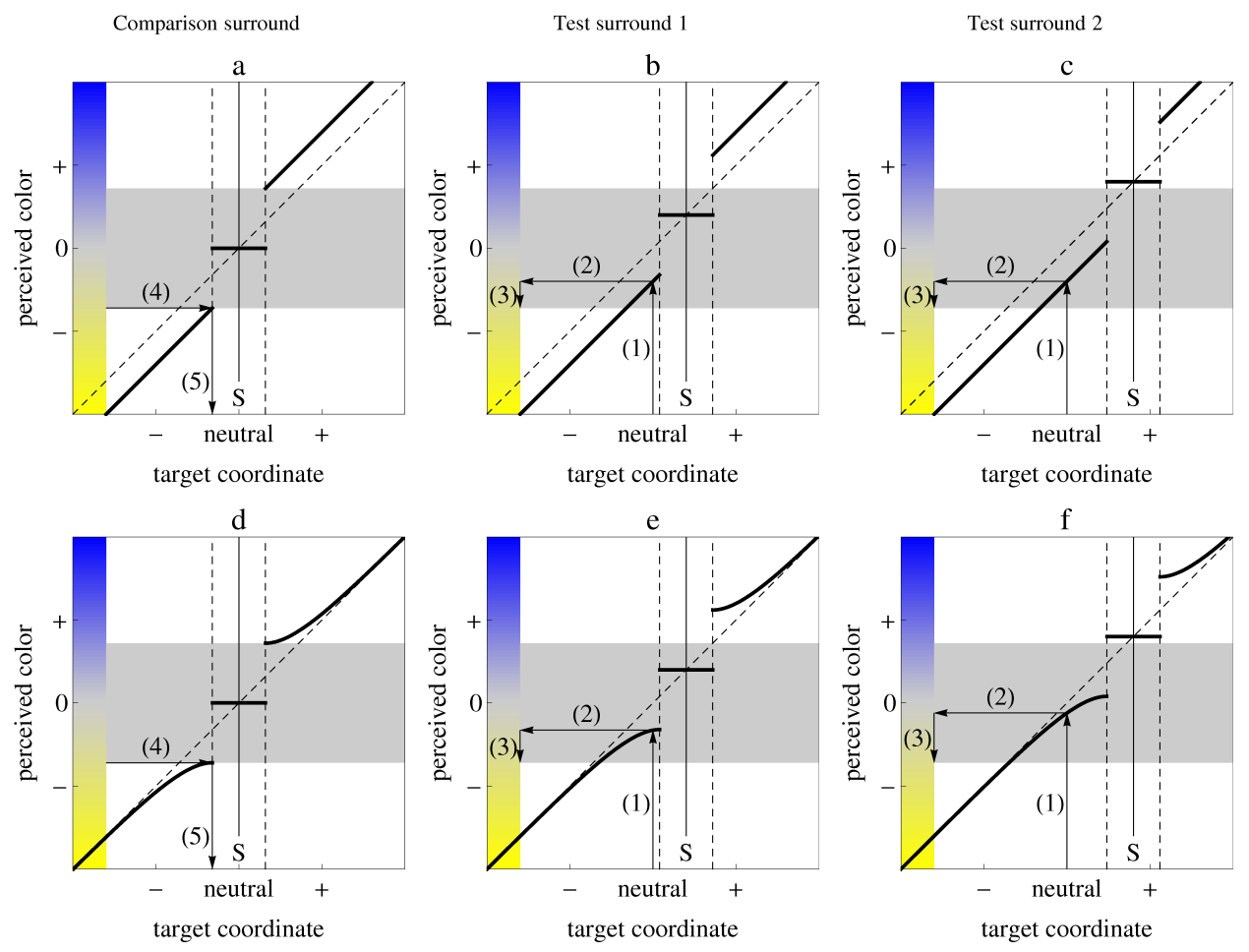

Figure 12: Derivation of predictions for matching experiments such as those of Cornelissen and Brenner (1991) and Bosten and Mollon (2007), where a nominally gray target embedded in a coloured surround is matched by a target presented in a grey comparison surround. All examples consider target colours on a line in colour space from yellow to blue going through the colour of the surround. The top panels show how perceived colour (vertical axis) should depend on the chromaticity of the target (horizontal axis) for a grey surround (panel a), a moderately saturated blue surround (panel b) and a more strongly saturated blue surround (panel c), according to the direction hypothesis in combination with the constant size hypothesis. The solid vertical line indicates the coordinate of the surround, and the dashed vertical ones indicate threshold in each direction. Below threshold the target is perceived as equal to the surround. Neglecting this singular point, the grey region indicates the set of missing colour (along the vertical axis) for the grey surround. In panels (b) and (c) the grey region is copied from panel (a). That is, it represents the set of missing colour in the grey surround, not those in the blue surrounds. Arrow (2) points to the predicted perceived colour of the nominally grey target in the blue surrounds. This colour is identical for both of the blue surrounds and falls within the set of missing colours for the grey surround. Thus, subjects can be expected to choose the closest realisable colour in the grey comparison surround, which is pointed to by arrow (3). Arrows (4) and (5) in panel (a) show the target chromaticity in the grey surround predicted to produce this colour impression. As can be seen, it is a yellow target which is just discriminable against the grey comparison surround. This result is independent of the saturation of the surround. Surprisingly, the same result can be deøed based on the inverse size hypothesis (bottom panels). Here, the saturation of the yellowness induced into the target in the blue surrounds decreases with the saturation of the surround, but the closest realisable match in the grey comparison surround remains the same. 


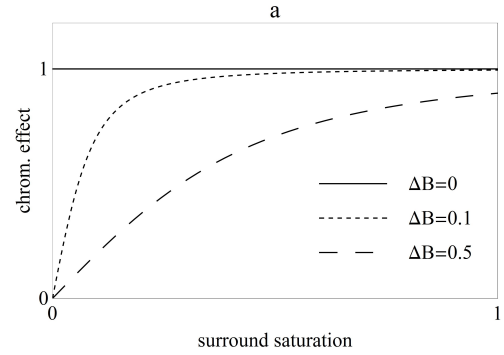

surround saturation

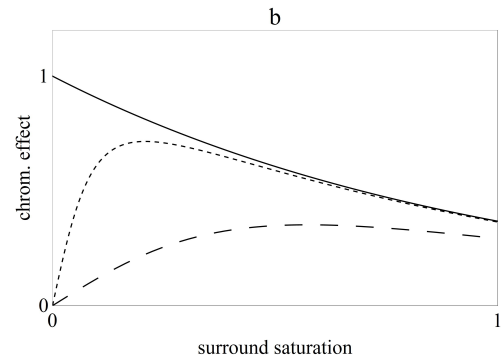

Figure 13: Predicted chromatic component of the simultaneous contrast effect induced into a nominally grey target as a function of surround saturation for different brightness offsets $\Delta B$ between target and surround. Panel (a) shows predictions based on the constant size hypothesis and panel (b) shows predictions based on the inverse size hypothesis. Note that when there is a brightness difference between the target and the surrounds, the chromatic component of the predicted effect can increase with surround saturation even when the constant size effect or the inverse size effect - rather than Kirschmann's fourth law - are generally valid. 
are exactly equal. Even slight deviations may produce misleading results.

Caveat E: Layered colour impressions Several authors have pointed to a role of perceptual transparency in colour illusions (Adelson, 1993, 2000; Anderson, 1997; Anderson \& Winawer, 2008; Wollschläger \& Anderson, 2009; Kingdom, Blakeslee, \& McCourt, 1997; Kingdom, 2011). Importantly, the layered colour impressions associated with perceptual transparency will also be evoked by simple centre-surround stimuli with a uniform surround, when target-surround contrast is low (Masin \& Idone, 1981; Brenner \& Cornelissen, 1991; Mausfeld, 1998; Ekroll et al., 2002, 2004). When such layered colour impressions are evoked in targets embedded in a uniform surround, one of the layers is perceived to be coextensive with the target, while the other extends across both target and surround. Our hypotheses are intended to describe the colour appearance of the colour component which is perceived as coextensive with the target. According to our informal observations, the other layer is typically perceived to have the same colour as the surround. The primary evidence for the inverse size hypothesis stems from our study of the gamut expansion effect (Faul et al., 2008). In this case, it is plausible that the perceived colour of the background layer did not influence the subjects' settings much, since the surround was achromatic. If the surround is chromatic, on the other hand, as in the studies of Brenner and Cornelissen (1991) and Bosten and Mollon (2007), the background layer may influence subjects's settings more easily because it is perceived as chromatic. By way of example, assume that the inverse size hypothesis is true and consider a nominally grey target embedded in desaturated red surround. Since target-surround contrast is low, the perceived colour of the target has two layers. The layer coextensive with the target only should appear greenish, while the background layer should appear reddish. Contrast this with a situation where the red surround is highly saturated. In this case, there will be no perceptual transparency, because the target-surround contrast is large, and the amount of induced greenness will be less because of the inverse size law. If, however, a subjects' judgement is influence by the reddish background component, which is only present in the former case, this may neutralise or even reverse the dependence on contrast posited by the inverse contrast law.

Caveat F: Equating contrast/opacity If the constant size hypothesis or inverse contrast hypothesis is true, matching the colour appearance of two targets embedded in different surrounds will involve cases where the target-surround contrasts are very different for the two targets. If subjects experience this as disturbing and modify their settings slightly in the direction making the contrasts more similar, this 
will produce results suggestive of Kirschmann's fourth law. Subjects may bias their settings in this direction for two reasons. First, they may experience the mere difference in perceived contrast as disturbing. Second, the perceived opacity of the target seems to depend on target-surround contrast. While low target-surround contrast produce impressions of perceptual transparency (Masin \& Idone, 1981; Ekroll et al., 2004), high ones produce opaque colour impressions. Thus, subjects may bias their settings towards Kirschmann's fourth law if the try to minimise the perceived difference in opacity.

Discussion of the available empirical findings The investigation of Cornelissen and Brenner (1991) includes various viewing conditions in which the influence of temporal adaptation can be expected to differ. Among these conditions, their 'normal viewing conditon' can be expected to involve less confounding influence of adaptation than the others. Their data from this condition, which are most relevant for present purposes, are replotted in Fig. 14. The data stem from different subjects and differnt hue conditions, but since all of their datasets from the 'normal viewing condition' - read from their figures $2,3,5$ and 6 - were generally rather similar, they are plotted together here.

On both axes in Fig. 14, the unit of measurement is Euclidian distance from the achromatic point in the CIE xy-diagram. The measured simultaneous contrast effect do exhibit a tendency to increase with surround saturation, in accordance with Kirschmann's fourth law. Two aspects of the data are noticeable, though. First, the effect is generally very small. The mean across all data points, represented by the solid horizontal line in Fig. 14 was 0.012 . Second, the slope of the linear regression shown as a dashed line, was very close to zero (0.047). Thus, in absolute terms, the effect is virtually constant across saturation levels. In this experiment, a comparision surround of the same nominally gray colour as the test target was used. Thus, as explained above in the discussion of Caveat $\mathbf{C}$, both of our size hypotheses make the same prediction, namely that the settings should be constant at a positive value so small that the comparison target is just discriminable from the comparison surround. The data shown in Fig. 14 are in rather good agreement with this prediction. The slight deviations from flatness is easily attributable to a residual influence of temporal adaptation.

The data presented by Bosten and Mollon (2007) obtained under virtually identical conditions are in very good agreement with the findings of Cornelissen and Brenner (1991). Their data are also essentially flat, and the size of the effect is comparable (slightly less, it seems) when measured as distance in the CIE xy-diagram. The smallest surround saturations used by Bosten and Mollon are smaller than those investigated by Cornelissen and Brenner (1991), and in this region, one can observe a decrease in the effect as surround saturation tends 


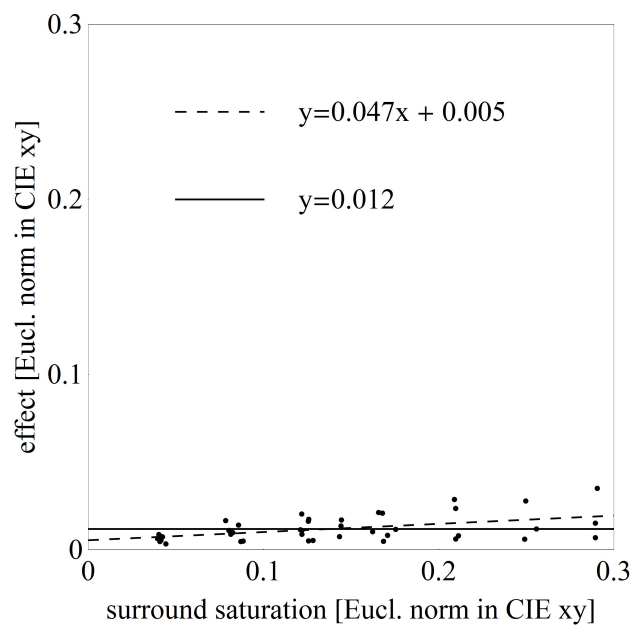

Figure 14: Data replotted from Cornelissen and Brenner (1991), showing how the measured simultaneous contrast effect induced into a grey surround depends on the saturation of the surround. Both the saturation of the surround and the simultaneous contrast effect is given as distance from the grey point in the CIE xy-diagram. A linear regression (dashed line) indicates but a very slight increase in the effect with target saturation. Note also that the average effect (solid line) is rather small (0.012 units in the CIE xy diagram). 
towards zero. It is not unlikely that this deviation from flatness occurs because it becomes difficult to discriminate the test target against the test surround at the lowest saturation levels (see the discussion of Caveat C above and Fig. 11). Bosten and Mollon's (2007) study includes a further condition, in which the comparison surround was black rather than grey. As predicted by our hypotheses (see discussion of Caveat $\mathbf{C}$ above), the simultaneous contrast effects measured in this condtion were larger. The data from this condition are also of some interest because, in this case, the constant size hypothesis and the inverse size hypothesis make different predictions. The data were essentially flat, which may be taken to favor the constant size hypothesis. However, the constant rather than decreasing data curve may well be an artifact due to Caveats $\mathbf{A}, \mathbf{E}$ or $\mathbf{F}$.

The relevant data from Kinney's (1962) study (read from her tables on pages 522-3) are replotted in Fig. 15. As can be seen, the data curves are again approximately flat, with a very slight positive slope for the blue and green surrounds. Note that in this experiment, the luminance of the surround was 1.2 times that of the target, hence Caveat D should be considered. Caveat A may also be relevant here. Note that the overall size of the measured effect is larger than in Cornelissen and Brenner's (1991) study (see Fig. 14). As explained above in connection with Caveat $\mathbf{C}$, this is to be expected based on our hypotheses because she used a black comparison surround rather than a grey one.

The data of Valberg (1974) document a clear increase in the measured effect with surround saturation. In his experiment, though, each eye adapted separately to the two surrounds, and long presentation times $(210 \mathrm{sec})$ were used. Therefore, the influence of adaptation may account for his results (see Caveat $\mathbf{A}$ above).

The reader may obtain a crude idea about how the colour appearance of a grey target depends on the saturation of the surround by inspecting the targets in the middle column in Fig. 6. Considering the vicissitudes of colour reproduction and that different observers may differ in their susceptibility to simultaneous contrast (Thouless, 1932; Ekroll \& Faul, 2009; Ekroll et al., 2011; Bosten \& Mollon, 2010), informal observations made with this demonstration should be taken with a grain of salt, but some tentative observations may nevertheless be of interest. First, the variation in the colour appearance within each chromatic half-axis (the four yellowish targets on top and the four bluish ones on the bottom) is clearly much less dramatic than the variation in the colour appearance of the corresponding surround colours, in agreement with the empirical results shown in Figs. 14 and 15. Second, the rather small variations in the colour appearance of the targets having the same perceived hue is rather difficult to describe. With decreasing surround saturation, the targets appear perhaps 'weaker' or less salient, 
a) red surrounds

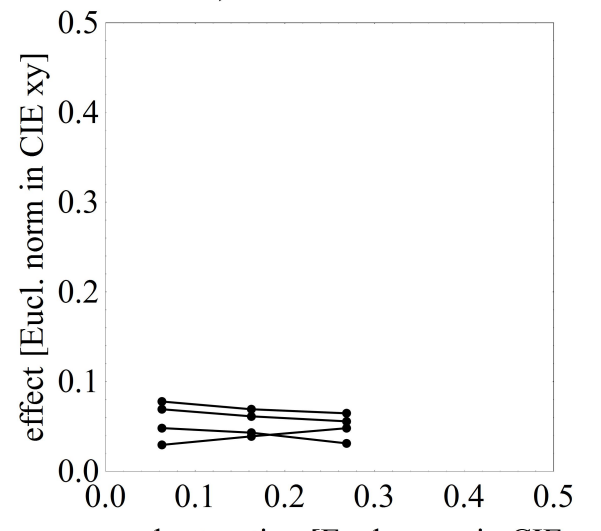

surround saturation [Eucl. norm in CIE xy]

\section{c) green surrounds}

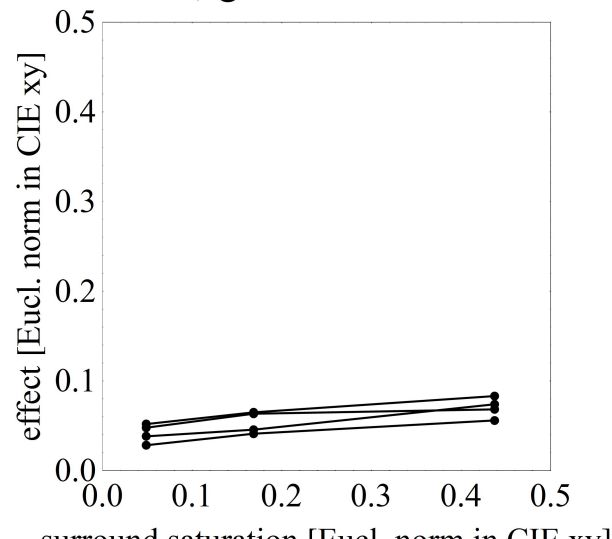

b) yellow surrounds

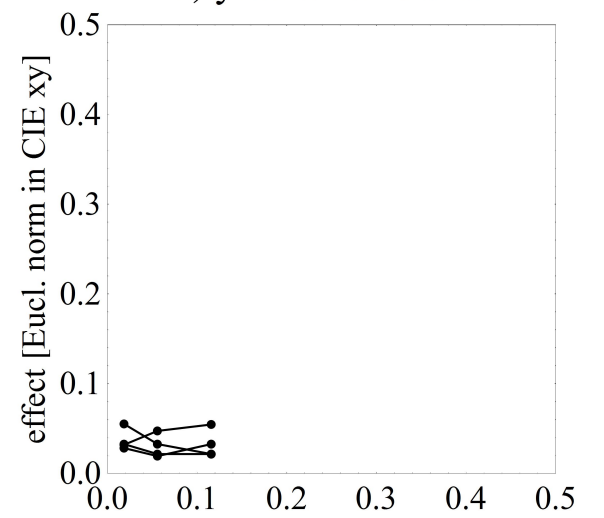

surround saturation [Eucl. norm in CIE xy]

d) blue surrounds

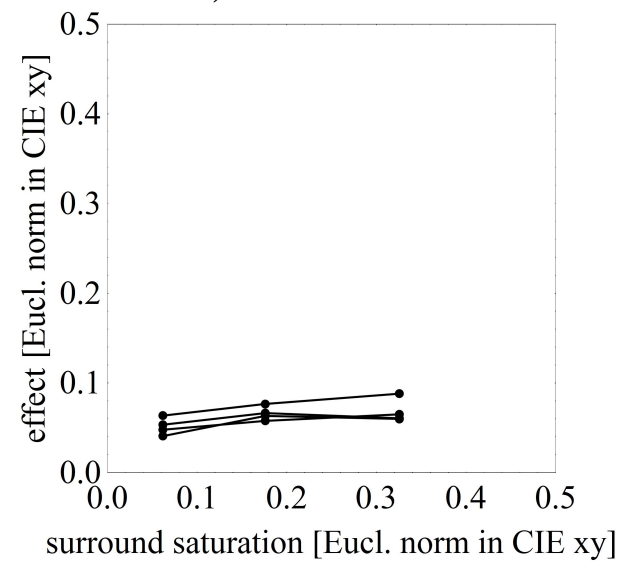

Figure 15: Plots of Kinney's (1962) data on how the simultaneous contrast effect depend on the saturation of the surround. Axes are the same as in Fig. 14. Each data curve shows the results for one of the five subjects. An increase in the effect with surround saturation is not indicated by the data obtained with the red and yellow surround, and the increase suggested by the data obtained with the green and blue surrounds is but very slight. 
but the conventional description, according to which they should appear less 'saturated' seems inappropriate, because - at least according to our phenomenal experience - there is no variation in perceived grey content. Variations in colourfulness are also difficult to pin down conclusively, and in a certain sense, the targets appear to have much the same colour, while it is also clear that they appear different. These differences may perhaps be better described as differences in the degree of transparency/opacity, 'thinness' or salience of the targets than as differences in saturation. Note also that Caveats A,C,E and $F$ must be taken into account when interpreting what can be observed in this demonstration.

In summary, when the abovementioned caveats are taken into account, the available evidence discussed in this section is compatible both with constant size hypothesis and the inverse contrast hypothesis. Viewed in conjunction with the evidence from our study of the gamut expansion effect (Faul et al., 2008), where presumably neither of the abovementioned Caveats A-F apply, the inverse contrast hypothesis may even seem more plausible. A final conclusion on this issue must however await the results from further experiments addressing the potential caveats more directly. Note, however, that even if the data from the studies of Cornelissen and Brenner (1991), Bosten and Mollon (2007) and Kinney (1962) are taken at face value, the increase in the effect with surround saturation is but very slight, as can be seen in Figs. 14 and 15. Thus, even if Kirschmann's fourth law may be valid on this literal reading of the data, we may safely conclude that they are approximated rather well by the constant size hypothesis.

\section{Discussion}

In this paper we have shown that new hypotheses challenging the traditional complementarity law and Kirschmann's fourth law of simultaneous contrast provide a unified framework for understanding many poorly understood and seemingly unrelated phenomena of colour appearance, namely

- the gamut expansion effect (Brown \& MacLeod, 1997),

- the 'missing colours' phenomenon (Ekroll et al., 2004),

- the convergence paradox (Ekroll et al., 2002),

- the rotation of constant hue loci (Hansen et al., 2007) and

- Kirschmann's third law (Kirschmann, 1891; Gordon \& Shapley, 2006)

We have also argued that the universally presumed validity of the complementarity law and Kirschmann's fourth law can be understood as resulting from the failure to take various confounding factors into account 
when interpreting empirical data. The most prominent confounding factor is the influence of temporal von Kries adaptation, which does obey the complementarity law and Kirschmann's fourth law and thus may distort empirical measurements accordingly in many situations. Our simple hypothesis of how von Kries adaptation and simultaneous contrast act together has some particularly interesting properties. First, it implies that asymmetric colour matching experiments involving conditions where temporal adaptation is in steady state, are not, as traditionally assumed (Whittle, 1994), particularly well-suited for studying simultaneous contrast, but rather particularly ill-suited. This is because when adaptation is complete, the simultaneous contrast effect will cancel out of the matching equation. Second, our hypothesis also explains why asymmetric matching is typically subjectively difficult when side-by-side displays (moderate influence of adaptation) are used, but typically subjectively easy when haploscopically superimposed displays (maximal influence of adaptation) are used (Whittle, 1994).

We have argued that when sources of artifact are taken into consideration, the evidence for Kirschmann's fourth law is not as strong as the literature may seem to suggest and that the available evidence is also compatible with the constant size hypothesis or maybe even the inverse size hypothesis. At present, though, it may be most appropriate to conclude that while these alternatives to Kirschmann's fourth law appear viable and interesting enough to warrant serious consideration, the presently available evidence does not allow for a definite conclusion on this issue. It is therefore of interest to consider how critically the different predictions discussed in this paper depend on it. The critical ingredient in accounting for the 'missing colours' phenomenon, the convergence paradox and the rotation of constant hue lines is the step discontinuity which follows from the direction hypothesis only if the constant size or the inverse size hypothesis is true. Thus, if the step discontinuity is postulated directly, one could dispose of the size hypotheses and obtain essentially the same predictions for these phenomena. The step discontinuity plays no role in the prediction of Kirschmann's third law. Here, the essential predictions would break down when the size of the effect $s$ is proportional to or a positively acceleration function of the target-surround difference $\Delta$, but are otherwise preserved. That is, an increasing function is possible as long as it is negatively accelerated.

With regard to Kirschmann's third law, our hypotheses make predictions which are virtually indistinguishable from those made by Gordon and Shapley's (2006) model. Thus, their empirical data are equally compatible with either approach. Our hypotheses, though, seem to be of more general applicability. While it is difficult to see how Gordon and Shapley's model can be extended to account for the gamut 
expansion effect, the 'missing colours' phenomenon, the convergence paradox, or the rotation of constant hue lines, our hypotheses account parsimoneously and naturally for all of these phenomena. Furthermore, different from Gordon and Shapley's model our hypotheses also predict the brightness effect observable in Fig. 10.

According to Gordon and Shapley's explanation, chromatic simulaneous contrast decreases with the brightness difference between target and surround because brightness contrast inhibits simultaneous colour contrast. A notion implicit in this explanation is that chromatic simultaneous contrast first occurs in a chromatic channel and is only subsequently inhibited when the information from the chromatic and brightness channels are combined. From the perspective of our hypothesis, simultaneous contrast operates on a colour signal consisting of the full colour signal (chromatic and brightness signals) from the very start. Thus, even though Gordon and Shapley's and our predictions are essentially the same for Kirschmann's third law, our approaches entail different perspectives regarding the stage in the visual process at which the simultaneous contrast occurs. In Gordon and Shapley's account, things are simple in each single channel and become more complicated when information from the channels are combined, while in our approach, the simple picture reveals itself in three-dimensional colour space but appears complicated when it is projected onto any single axis.

\section{Conclusions}

We have shown that several seemingly unrelated aspects of perceived colour can be parsimoniously accounted for if one assumes a) that the direction of the simultaneous contrast effect is equal to that of the colour vector pointing from surround to target and b) that the size of the effect is constant or even decreases with the difference between target and surround.

\section{Acknowledgements}

We are greatly indebted to two anonymous reviewers for important suggestions.

\section{References}

Adelson, E. H. (1993). Perceptual organization and the judgment of brightness. Science, 262(5142), 2042-4. 
Adelson, E. H. (2000). Lightness perception and lightness illusions. In M. Gazzaniga (Ed.), The new cognitive neurosciences, 2 edition (p. 339-351). Cambrigde, MA: MIT Press.

Anderson, B. L. (1997). A theory of illusory lightness and transparency in monocular and binocular images: the role of contour junctions. Perception, 26, 419-452.

Anderson, B. L., \& Winawer, J. (2008, 7). Layered image representations and the computation of surface lightness. $J$. Vis., 8(7), 1-22. Available from http://journalof vision . org $/ 8 / 7 / 18 /$

Bosten, J., \& Mollon, J. (2007). Kirschmann's fourth law. Perception, 36 ECVP Abstract Supplement, 190.

Bosten, J., \& Mollon, J. (2010). Is there a general trait of susceptibility to simultaneous contrast? Vision research, 50(17), 1656-1664.

Brenner, E., \& Cornelissen, F. W. (1991). Spatial interactions in color vision depend on distances between boundaries. Naturwissenschaften, 78, 70-73.

Brown, R. O. (2003). Backgrounds and illuminants: the yin and yang of color constancy. In R. Mausfeld \& D. Heyer (Eds.), Colour: Mind and the physical world (p. 247-271). Oxford University Press.

Brown, R. O., \& MacLeod, D. I. A. (1997). Color appearance depends on the variance of surround colors. Curr Biol, $7(11), 844-9$.

Burgh, P., \& Grindley, G. (1962). Size of test patch and simultaneous contrast. The Quarterly Journal of Experimental Psychology.

Cataliotti, J., \& Becklen, R. (2007). Single dissociation between lightness contrast effects. Perception, 36 ECVP Abstract Supplement, 79.

Chichilnisky, E. J., \& Wandell, B. A. (1995). Photoreceptor sensitivity changes explain color appearance shifts induced by large uniform backgrounds in dichoptic matching. Vision Res, 35(2), 239-54.

Cornelissen, F. W., \& Brenner, E. (1991). On the role and nature of adaptation in chromatic induction. In B. Blum (Ed.), Channels in the visual nervous system: Neurophysiology, psychohysics and models (p. 109-123). London and Tel 
Aviv: Freund Publishing House, Ltd.

Ekroll, V. (2005). On the nature of simultaneous colour contrast. Philosophische Fakultät der Christian-Albrechts-Universität zu Kiel. Available from http://eldiss.uni-kiel.de/macau/receive/ dissertation_diss_00001459

Ekroll, V., \& Faul, F. (2009). A simple model describes large individual differences in simultaneous colour contrast. Vision Research, 49(18), 2261 - 2272. Available from http:// wWw. sciencedirect.com/science/article/B6TOW -4WKK1H8-1/2/5bb1a0794cc62301e5649ca232dd8599

Ekroll, V., Faul, F., \& Niederée, R. (2004). The peculiar nature of simultaneous colour contrast in uniform surrounds. Vision Res, 44(15), 1765-1786. Available from http://dx.doi.org/10.1016/j.visres.2004.02.009

Ekroll, V., Faul, F., Niederée, R., \& Richter, E. (2002). The natural center of chromaticity space is not always achromatic: a new look at color induction. Proc Natl Acad Sci U S A, 99(20), 13352-13356. Available from http:// dx.doi.org/10.1073/pnas. 192216699

Ekroll, V., Faul, F., \& Wendt, G. (2011). The strengths of simultaneous colour contrast and the gamut expansion effect correlate across observers: evidence for a common mechanism. Vision Res, 51(3), 311-322. Available from http://dx.doi.org/10.1016/j.visres.2010.11.009

Faul, F., Ekroll, V., \& Wendt, G. (2008). Color appearance: The limited role of chromatic surround variance in the "gamut expansion effect". J Vis, 8(3), 30.1-3020. Available from http://dx.doi.org/10.1167/8.3.30

Gelb, A. (1929). Die „Farbenkonstanz” der Sehdinge. In A. Bethe, G. von Bergman, G. Embden, \& A. Ellinger (Eds.), Handbuch der der normalen und pathologischen Physiologie (p. 594-687). Berlin: Springer. (Bd. 12, 1. Hälfte. Rezeptionsorgane II)

Gordon, J., \& Shapley, R. (2006). Brightness contrast inhibits color induction: evidence for a new kind of color theory. Spat Vis, 19(2-4), 133-146.

Hansen, T., Walter, S., \& Gegenfurtner, K. R. (2007). Effects of spatial and temporal context on color categories and color constancy. J Vis, 7(4), 2. Available from http:// 
dx.doi.org/10.1167/7.4.2

Helmholtz, H. von (Ed.). (1911). Handbuch der Physiologischen Optik. Hamburg und Leipzig: Verlag von Leopold Vos.

Helson, H., \& Michels, W. C. (1948). The effect of chromatic adaptation on achromaticity. Journal of the Optical Society of America, 38(12), 1025-1032.

Hering, E. (1887). Ueber die Theorie des simultanen Contrastes von Helmholtz: II. Der Contrastversuch von H. Meyer und die Versuche am Farbenkreisel. Pflügers Archiv für Physiologie, 41, 1-29.

Hildreth, E. C. (1949). Lectures on visual psychology. Chicago: Professional Press.

Jameson, D., \& Hurvich, L. M. (1961). Opponent chromatic induction: Experimental evaluation and theoretical account. Journal of the Optical Society of America, 51(1), 46-53.

Kingdom, F. A. A. (2011, Apr). Lightness, brightness and transparency: a quarter century of new ideas, captivating demonstrations and unrelenting controversy. Vision Res, 51(7), 652-673. Available from http://dx.doi.org/ 10.1016/j.visres.2010.09.012

Kingdom, F. A. A., Blakeslee, B., \& McCourt, M. E. (1997). Brightness with and without perceived transparency: when does it make a difference? Perception, 26 (4), 493-506.

Kinney, J. A. S. (1962). Factors affecting induced color. Vision Research, 2, 503-525.

Kirschmann, A. (1891). Ueber die quantitativen Verhältnisse des simultanen Helligkeits- und Farben-Contrastes. Philosophische Studien, 6, 417-491.

Köhler, J. (1904). Der simultane Farben- und Helligkeitskontrast, mit besonderer Berücksichtigung des sog. Florkontrastes. Archiv für die gesamte Psychologie, 2, 1531-40.

Kries, J. von. (1905). Die Gesichtsempfindungen. In W. Nagel (Ed.), Handbuch der Physiologie des Menschen (Vols. 3, Physiologie der Sinne, p. 109-282). Braunschweig: Friedrich Vieweg und Sohn.

Masin, S. C., \& Idone, A. M. (1981). Studio sperimentale sulla percezione della trasparenza con figura e sfondo achromatici e omogenei. Giornale Italiano di Psicologia, 8, 265277.

Mausfeld, R. (1998). Color perception: From Grassman codes 
to a dual code for object and illumination colors. In W. G. K. Backhaus, R. Kliegl, \& J. S. Werner (Eds.), Color vision (p. 219-250). Berlin: De Gruyter.

Mausfeld, R. (2003). 'Colour' as part of the format of different perceptual primitives: the dual coding of colour. In R. Mausfeld \& D. Heyer (Eds.), Colour perception: Mind and the physical world (p. 381-429). Oxford University Press.

Metelli, F. (1970). An algebraic development of the theory of perceptual transparency. Ergonomics, 13, 59-66.

Meyer, H. (1855). Über Kontrast- und Komplementärfarben. Annalen der Physik, XCV, 170-171.

Mizokami, Y., \& Yaguchi, H. (2010). Perception of colorfulness influenced by chromatic variance in indoor environments. Journal of Light \& Visual Environment, 34 (2), 69-75.

Niederée, R., \& Mausfeld, R. (1997). Increment-decrement asymmetry in dichoptic matching with haploscopically superimposed backgrounds. Vision Res, 37(5), 613-6.

Perls, L. (1932). Die Erscheinungen des simultanen Kontrastes und der Eindruck der Feldbeleuchtung. Dissertation, Johann Wolfgang Goethe-Universität, Frankfurt am Main.

Richter, E. (2002). Modelle der Kontrastcodierung. Ph. D. thesis, Martin-Luther-Universität Halle-Wittenberg.

Rinner, O., \& Gegenfurtner, K. R. (2000). Time course of chromatic adaptation for color appearance and discrimination. Vision Res, 40(14), 1813-26.

Shepherd, A. J. (1997). A vector model of colour contrast in a cone-excitation colour space. Perception, 26 (4), 455-470.

Shepherd, A. J. (1999). Remodelling colour contrast: implications for visual processing and colour representation. $V i$ sion Research, 39(7), 1329-1345.

Thouless, R. (1932). Individual differences in phenomenal regression. British Journal of Psychology. General Section, 22(3), 216-241.

Valberg, A. (1974). Color induction: dependence on luminance, purity, and dominant or complementary wavelength of inducing stimuli. J Opt Soc Am, 64(11), 1531-40.

Valberg, A., \& Lange-Malecki, B. (1990). "Colour constancy" in Mondrian patterns: a partial cancellation of physical chromaticity shifts by simultaneous contrast. Vision Research, 
$30(3), 371-380$.

Vladusich, T., Lucassen, M. P., \& Cornelissen, F. W. (2007). Brightness and darkness as perceptual dimensions. PLoS Comput Biol, 3(10), e179. Available from http://dx.doi . org/10.1371/journal.pcbi.0030179

Walls, G. L. (1960). Land! Land! Psychological Bulletin, 57, $29-48$.

Werner, J. S., \& Walraven, J. (1982). Effect of chromatic adaptation on the achromatic locus: the role of contrast, luminance and background color. Vision Res, 22, 929-943.

Whittle, P. (1994). The psychophysics of contrast brightness. In A. L. Gilchrist (Ed.), Lightness, brightness and transparency (p. 35-110). Lawrence Erlbaum Associates.

Whittle, P. (2003). Contrast colours. In R. Mausfeld \& D. Heyer (Eds.), Colour perception: Mind and the physical world (p. 115-138). Oxford University Press.

Whittle, P., \& Challands, P. D. C. (1969). The effect of background luminance on the brightness of flashes. Vision Research, 9, 1095-1110.

Wollschläger, D., \& Anderson, B. L. (2009, Mar). The role of layered scene representations in color appearance. Curr Biol, 19(5), 430-435. Available from http://dx.doi.org/10 .1016/j.cub. 2009.01.053

Wuerger, S. M. (1996). Color appearance changes resulting from iso-luminant chromatic adaptation. Vision Research, $36(19), 3107-3118$. 